\title{
Pricing vulnerable options with variable default boundary under jump-diffusion processes
}

\author{
Qing Zhou ${ }^{1 *}$, Qian Wang ${ }^{1}$ and Weixing $\mathrm{Wu}^{2}$
}

"Correspondence:
zqleii@bupt.edu.cn
'School of Science, Beijing
University of Posts and
Telecommunications, Beijing, China
Full list of author information is
available at the end of the article

available at the end of the article

\begin{abstract}
For the pricing of vulnerable options, we improve the results of Klein and Inglis [Journal of Banking and Finance] and Tian et al. [The Journal of Futures and Markets], considering the circumstances in which the writers of options face financial crisis. Our pricing model faces the risks of default and the occasional impact experienced by the underlying assets and counterparty's assets. The correlation between the option's underlying assets and the option writer's assets is clearly modeled. Asset prices are driven by the jump-diffusion processes of two related assets. Furthermore, we consider a variable default boundary (VDB) based on the option's potential debt and the option writer's other liabilities. In case financial distress happens, the payout rate is connected to the option writer's assets. Through the Taylor expansion, we derive an approximate explicit valuation for vulnerable options.
\end{abstract}

MSC: $60 \mathrm{H} 10 ; 60 \mathrm{~J} 75 ; 60 \mathrm{H} 30 ; 90 \mathrm{G} 20 ; 90 \mathrm{G} 40$

Keywords: Credit risk; Default; Jump-diffusion; Pricing; Vulnerable option

\section{Introduction}

In the 1990s and earlier, financial market participants did not pay enough attention to counterparty risk. On account of the impact of the global financial crisis, more and more investors and researchers have begun to consider the influence of counterparty risk on the company's credit risk. Now many financial institutions trade their derivatives with counterparties and other institutions in the over-the-counter (OTC for short) markets. Due to system defects used for OTC market transactions as well as ineffective regulation, credit risk becomes the major risk threatening the stability of the entire financial system. Thus, accurately pricing the various options to effectively resist financial crises is urgent in the OTC markets. The standard option pricing formulas $[1,2]$ do not focus on counterparty risk. Subsequently, some literatures begin to pay attention to the pricing of vulnerable options and consider counterparty risk (see [3-13]).

Specifically, Johnson and Stulz [3] think that the option is the writer's sole liability. When the counterparty defaults, the option holder will receive all the assets of the option writer. Hull and White [5] extend the model, assume that the counterparty has other liabilities, the payout rate is exogenous, and the option holder will obtain part of the option's intrinsic value when the counterparty defaults, thereby deriving the vulnerable option's pricing

(c) The Author(s) 2018. This article is distributed under the terms of the Creative Commons Attribution 4.0 International License (http://creativecommons.org/licenses/by/4.0/), which permits unrestricted use, distribution, and reproduction in any medium, provided you give appropriate credit to the original author(s) and the source, provide a link to the Creative Commons license, and indicate if changes were made. 
formula. Jarrow and Turnbull [6] propose a risk neutral evaluating mechanism of derivative securities exposed to default risk. Klein [7] provides an analytical solution of the European vulnerable option's price, taking into account the interrelationship between the option writer's asset and the underlying asset. They also assume that the option writers have other liabilities, and when the counterparty defaults, some of the nominal claims will be paid.

Recently, Klein and Inglis [8] and Hung and Liu [9] have extended the model of [7] to obtain closed-form solutions of European vulnerable options facing interest rate risk and financial crisis, and to price vulnerable options in incomplete markets, respectively. Klein and Inglis [10] extend the results of [3] and [7] through incorporating the VDB that relies on both the potential liabilities of the options and the other liabilities of the option writers. If a financial distress occurs, the payout rate is associated with the asset of the option writer, and the correlation between the underlying asset and the option writer's asset is explicitly modeled. They get an approximate analytical formula for vulnerable options. In [11], the pricing model of vulnerable options not only faces default risk but also faces the occasional impact of the underlying asset and the counterparty's assets. These two types of assets are related. The asset price dynamics follow the jump-diffusion processes. The jump is divided into two parts: the trait part of each asset price and the part of the system that affects the price of all assets. In [12], when the underlying asset follows a stochastic volatility model, Yang et al. use asymptotic analysis to obtain an approximate analytical pricing formula for vulnerable options. Yoon et al. [13] use the Double Merlin Transform under constant and random (Hull-White) interest rates to get analytical pricing formulas for each interest rate case so that vulnerable option's value can be accurately and effectively calculated. Through a Markov modulation mechanism switching method to simulate various economic conditions and giving the dynamics of the assets values by two related jump-diffusion processes, Niu et al. [4] study the pricing of vulnerable European options. By Laplace transforms, they obtain an analytical solution of the price of vulnerable options.

This paper will extend the results of [10] and [11] for valuing vulnerable options when the option writer suffers the financial crisis. As in [11], the asset prices are driven by jumpdiffusion processes. The jump is divided into two parts: the trait part of each asset price and the part of the system that affects the price of all assets. Following Klein and Inglis [10], we assume that there are interrelations between the option's underlying asset and the option writer's assets, and the option writers have other liabilities. The value growth of the option itself may also give rise to financial distress. The default barrier is divided into a stochastic part that measures the option's potential payoff and the fixed part that represents the option writer's other liabilities. Furthermore, we make the assumption that only variations in the value of the asset underlying the option or the writer's assets can cause financial distress. By the Taylor expansion, we derive an approximate explicit valuation for vulnerable options.

We proceed as follows. In Sect. 2, a jump-diffusion model incorporating a variable default boundary (VDB for short) is proposed, and we obtain an approximate explicit solution of vulnerable European option. We present the detailed technical calculations in the Appendix. 


\section{Pricing vulnerable options}

\subsection{The model}

Let $(\Omega, \mathcal{F}, \mathbb{Q})$ be a complete probability space and $r$ be the instantaneous riskless rate. Jump processes are given to describe the discontinuous changes of prices. We will give the following assumptions underlying our model for vulnerable option. One can see references [10] and [11] for the detailed description.

Assumption 1 Suppose that $S_{t}$ denotes the price of the underlying asset of the option. The dynamics of $S_{t}$ are presented as follows:

$$
\frac{d S_{t}}{S_{t-}}=\left(r-k_{S} \lambda_{S}^{*}\right) d t+\sigma_{S} d W_{t}^{(1)}+\left(\mathrm{e}^{Z_{t-}^{(1)}}-1\right) d M_{t}^{(1)}
$$

where $W_{t}^{(1)}$ is a standard Brownian motion on $(\Omega, \mathcal{F}, \mathbb{Q})$, and $\sigma_{S}$ denotes the volatility. $M_{t}^{(1)}$ represents a Poisson process, which models the jumps of the underlying asset price. $\lambda_{S}^{*}$ denotes the jump intensity of $M_{t}^{(1)} \cdot M_{t}^{(1)}$ and $\lambda_{S}^{*}$ both consist of two parts,

$$
\begin{aligned}
& M_{t}^{(1)}=N_{t}^{(1)}+N_{t}, \\
& \lambda_{S}^{*}=\lambda_{S}+\lambda,
\end{aligned}
$$

where $N_{t}^{(1)}$ models the individual impact on the asset price, and $N_{t}$ models common shocks that also have influence on the counterparty's asset. $N_{t}$ and $N_{t}^{(1)}$ are both Poisson processes with intensities $\lambda$ and $\lambda_{S}$, respectively. We assume that they are independent. $Z_{t}^{(1)}$ denotes the underlying asset's jump amplitude when the jump happens. We suppose that $Z_{s}^{(1)}$ and $Z_{t}^{(1)}$ are independent and have the same distribution when $s \neq t . k_{S}=\mathbb{E}\left[\mathrm{e}_{t}^{Z_{t}^{(1)}}\right]-1$ represents the average jump percentage of the price. We always assume that $k_{S}$ is finite and $Z_{t}^{(1)}$ is a normal random variable with expectation $\mu_{1}$ and variation $\sigma_{1}^{2}$. Then $k_{S}=\mathrm{e}^{\mu_{1}+\frac{1}{2} \sigma_{1}^{2}}-1$.

Assumption 2 Suppose that $V_{t}$ denotes the value of the option writer's asset. The dynamics of $V_{t}$ are presented as follows:

$$
\frac{d V_{t}}{V_{t-}}=\left(r-k_{V} \lambda_{V}^{*}\right) d t+\sigma_{V} d W_{t}^{(2)}+\left(\mathrm{e}^{Z_{t-}^{(2)}}-1\right) d M_{t}^{(2)}
$$

where $W_{t}^{(2)}$ denotes a standard Brownian motion on $(\Omega, \mathcal{F}, \mathbb{Q})$, and $\sigma_{V}$ represents the volatility of the counterparty's asset. We assume that $\rho$ denotes the correlation coefficient of $W_{t}^{(1)}$ and $W_{t}^{(2)} \cdot M_{t}^{(2)}$ represents a Poisson process, which models the jumps of the asset price $V_{t} \cdot \lambda_{V}^{*}$ denotes the intensity of $M_{t}^{(2)} \cdot M_{t}^{(2)}$ and $\lambda_{V}^{*}$ both consist of two parts,

$$
\begin{aligned}
& M_{t}^{(2)}=N_{t}^{(2)}+N_{t}, \\
& \lambda_{V}^{*}=\lambda_{V}+\lambda,
\end{aligned}
$$

where $N_{t}^{(2)}$ is a Poisson process independent of $N_{t}^{(1)}$ and $N_{t} . N_{t}^{(2)}$ modeling the individual impact on the asset price has the intensity $\lambda_{V}$. $Z_{t}^{(2)}$ denotes the jump amplitude of the underlying asset when the jump happens. Suppose that $Z_{t}^{(2)}$ is distributed normally by $N_{1}\left(\mu_{2}, \sigma_{2}^{2}\right)$, and $Z_{t}^{(2)}$ and $Z_{s}^{(2)}$ are independently and identically distributed 
when $t \neq s$, where $N_{1}(\cdot, \cdot)$ denotes the one-dimensional normal distribution function. $k_{V}=\mathbb{E}\left[\mathrm{e}^{Z_{t}^{(2)}}\right]-1=\mathrm{e}^{\mu_{2}+\frac{1}{2} \sigma_{2}^{2}}-1$ denotes the mean percentage jump when the jump arrives. Furthermore, we assume that $\left(W_{t}^{(1)}, W_{t}^{(2)}\right), N_{t}, Z_{t}^{(1)}, N_{t}^{(1)}, Z_{t}^{(2)}$, and $N_{t}^{(2)}$ are independent.

In our model, we consider the related credit risk not only in the continuous section but also in the point process section. Regarding the continuous section, $W_{t}^{(1)}$ and $W_{t}^{(2)}$ have the correlation coefficient $\rho$. Regarding the point process section, relevance is reflected in the common market factors $N_{t}$.

Assumption 3 Default happens only at the maturity of the option $T$ when the threshold value $D^{*}+c_{T}$ is more than the value of the option writer's asset $V_{T}$, where $D^{*}$ denotes the value of the option writer's other liabilities, and $c_{T}=\left(S_{T}-K\right)^{+} . S_{T}$ denotes the underlying asset's price at the maturity, and $K$ denotes the excise price of the option.

Assumption 4 When the financial crisis happens, the option holder receives $(1-w)$ times the intrinsic value of the option at its maturity. $w$ denotes the percentage reduction of the option holder's nominal claim. We assume that $w=1-\frac{(1-\alpha) V_{T}}{D^{*}+c_{T}}$, where $\alpha$ denotes the deadweight costs of the financial crisis, and $\frac{V_{T}}{D^{*}+c_{T}}$ denotes the value of the option writer's assets which can be used to pay the claim represented as a percentage of the total claims at $T$.

Based on the methods used in [10] and [11], we get the approximate explicit valuation of the vulnerable options.

\subsection{Valuation of European vulnerable options}

Let $C^{*}$ denote the value of a vulnerable European calls, which can be presented as

$$
C^{*}=\mathrm{e}^{-r T} \mathbb{E}\left[\left(S_{T}-K\right)^{+}\left(I_{\left\{V_{T} \geq D^{*}+S_{T}-K\right\}}+\frac{(1-\alpha) V_{T}}{D^{*}+S_{T}-K} I_{\left\{V_{T}<D^{*}+S_{T}-K\right\}}\right)\right] .
$$

The first part of this equation is the standard expression for the payoff on a European call option when there is no financial crisis, i.e., $V_{T} \geq D^{*}+S_{T}-K$. The second part indicates that in case financial distress happens, i.e., $V_{T}<D^{*}+S_{T}-K$, the entire assets of the option writer can be allocated to the option holder and other creditors of the option writer. The rate $\frac{S_{T}-K}{D^{*}+S_{T}-K}$ represents the proportion of the amount that can be used to pay to the option holder.

Through the Itô formula, we have the following equalities:

$$
\begin{aligned}
& \ln S_{T}=\ln S_{0}+\left(r-\frac{1}{2} \sigma_{S}^{2}-k_{S} \lambda_{S}^{*}\right) T+\sigma_{S} W_{T}^{(1)}+\sum_{k=1}^{M_{T}^{(1)}} Z_{\tau_{k}^{(1)}}^{(1)}, \\
& \ln V_{T}=\ln V_{0}+\left(r-\frac{1}{2} \sigma_{V}^{2}-k_{V} \lambda_{V}^{*}\right) T+\sigma_{V} W_{T}^{(2)}+\sum_{k=1}^{M_{T}^{(2)}} Z_{\tau_{k}^{(2)}}^{(2)},
\end{aligned}
$$

where $\tau_{k}^{(i)}$ represents the $k$ th jump time of $M_{t}^{(i)}, i=1,2$, respectively. Suppose that $Z_{t}^{(i)}$ distributes normally by $N_{1}\left(\mu_{i}, \sigma_{i}^{2}\right), i=1,2$. Conditional on

$$
\mathcal{G}_{T}^{\left(n, n_{1}, n_{2}\right)}=\left\{N_{T}=n, N_{T}^{(1)}=n_{1}, N_{T}^{(2)}=n_{2}\right\},
$$


the total jump times of $S_{t}$ and $V_{t}$ are denoted by

$$
\begin{aligned}
& m_{1}=n+n_{1}, \\
& m_{2}=n+n_{2} .
\end{aligned}
$$

Obviously, $\left(\ln \frac{S_{T}}{S_{0}}, \ln \frac{V_{T}}{V_{0}}\right)$ is a bivariate normal random vector with the following numerical characteristics:

$$
\begin{aligned}
& M_{1}\left(m_{1}\right)=\mathbb{E}\left[\ln \frac{S_{T}}{S_{0}}\right]=\left(r-\frac{1}{2} \sigma_{S}^{2}-k_{S} \lambda_{S}^{*}\right) T+m_{1} \mu_{1}, \\
& M_{2}\left(m_{2}\right)=\mathbb{E}\left[\ln \frac{V_{T}}{V_{0}}\right]=\left(r-\frac{1}{2} \sigma_{V}^{2}-k_{V} \lambda_{V}^{*}\right) T+m_{2} \mu_{2}, \\
& \operatorname{Cov}\left(\ln \frac{S_{T}}{S_{0}}, \ln \frac{V_{T}}{V_{0}}\right)=\rho \sigma_{S} \sigma_{V} T .
\end{aligned}
$$

\section{Denote}

$$
\begin{aligned}
& \ln S_{T, m_{1}}=\ln S_{0}+\left(r-\frac{1}{2} \sigma_{S}^{2}-k_{S} \lambda_{S}^{*}\right) T+\sigma_{S} W_{T}^{(1)}+\sum_{k=1}^{m_{1}} \xi_{k}^{(1)}, \\
& \ln V_{T, m_{2}}=\ln V_{0}+\left(r-\frac{1}{2} \sigma_{V}^{2}-k_{V} \lambda_{V}^{*}\right) T+\sigma_{V} W_{T}^{(2)}+\sum_{k=1}^{m_{2}} \xi_{k}^{(2)},
\end{aligned}
$$

where $\xi_{k}^{(i)}$ are independent and distribute normally by $N\left(\mu_{i}, \sigma_{i}^{2}\right), i=1,2$. The probability space can be decomposed: $\Omega=\bigcup_{n=0}^{\infty} \bigcup_{n_{1}=0}^{\infty} \bigcup_{n_{2}=0}^{\infty} \mathcal{G}_{T}^{\left(n, n_{1}, n_{2}\right)}$ and $\mathcal{G}_{T}^{\left(i, i_{1}, i_{2}\right)} \cap \mathcal{G}_{T}^{\left(j, j_{1}, j_{2}\right)}=\emptyset$ for any $i \neq j, i_{1} \neq j_{1}$, and $i_{2} \neq j_{2}$.

Thus, $C^{*}$ can be rewritten as follows:

$$
\begin{aligned}
C^{*}= & \mathrm{e}^{-r T} \mathbb{E}\left[\left(S_{T}-K\right)^{+}\left(I_{\left\{V_{T} \geq D^{*}+S_{T}-K\right\}}+\frac{(1-\alpha) V_{T}}{D^{*}+S_{T}-K} I_{\left\{V_{T}<D^{*}+S_{T}-K\right\}}\right) I_{\{\omega \in \Omega\}}\right] \\
= & \mathrm{e}^{-r T} \sum_{n=0}^{\infty} \sum_{n_{1}=0}^{\infty} \sum_{n_{2}=0}^{\infty} \mathbb{E}\left[( S _ { T } - K ) ^ { + } \left(I_{\left\{V_{T} \geq D^{*}+S_{T}-K\right\}}\right.\right. \\
& \left.\left.+\frac{(1-\alpha) V_{T}}{D^{*}+S_{T}-K} I_{\left\{V_{T}<D^{*}+S_{T}-K\right\}}\right) I_{\left\{\omega \in \mathcal{G}_{T}^{\left(n, n_{1}, n_{2}\right)}\right\}}\right] \\
= & \sum_{n=0}^{\infty} \sum_{n_{1}=0}^{\infty} \sum_{n_{2}=0}^{\infty} \mathbb{Q}\left(N_{T}=n, N_{T}^{(1)}=n_{1}, N_{T}^{(2)}=n_{2}\right) \\
& \cdot \mathrm{e}^{-r T} \mathbb{E}\left[( S _ { T , m _ { 1 } } - K ) ^ { + } \left(I_{\left\{V_{T, m_{2}} \geq D^{*}+S_{T, m_{1}}-K\right\}}\right.\right. \\
& \left.\left.+\frac{(1-\alpha) V_{T, m_{2}}}{D^{*}+S_{T, m_{1}}-K} I_{\left\{V_{T, m_{2}}<D^{*}+S_{T, m_{1}}-K\right\}}\right)\right] \\
= & \sum_{n=0}^{\infty} \sum_{m_{1}=n m_{2}=n}^{\infty} \frac{(\lambda T)^{n}}{n !} \frac{\left(\lambda_{S} T\right)^{m_{1}-n}}{\left(m_{1}-n\right) !} \frac{\left(\lambda_{V} T\right)^{m_{2}-n}}{\left(m_{2}-n\right) !} \mathrm{e}^{-\lambda T-\lambda_{S} T-\lambda_{V} T} C_{m_{1}, m_{2}},
\end{aligned}
$$


where

$$
\begin{aligned}
C_{m_{1}, m_{2}}= & \mathrm{e}^{-r T} \mathbb{E}\left[( S _ { T , m _ { 1 } } - K ) ^ { + } \left(I_{\left\{V_{T, m_{2}} \geq D^{*}+S_{T, m_{1}}-K\right\}}\right.\right. \\
& \left.\left.+\frac{(1-\alpha) V_{T, m_{2}}}{D^{*}+S_{T, m_{1}}-K} I_{\left\{V_{T, m_{2}}<D^{*}+S_{T, m_{1}}-K\right\}}\right)\right] .
\end{aligned}
$$

Now, we divide $C_{m_{1}, m_{2}}$ into four parts for further calculation:

$$
C_{m_{1}, m_{2}}=\mathrm{e}^{-r T}\left[C_{1}\left(m_{1}, m_{2}\right)+C_{2}\left(m_{1}, m_{2}\right)+C_{3}\left(m_{1}, m_{2}\right)+C_{4}\left(m_{1}, m_{2}\right)\right]
$$

where $C_{1}\left(m_{1}, m_{2}\right), C_{2}\left(m_{1}, m_{2}\right), C_{3}\left(m_{1}, m_{2}\right)$, and $C_{4}\left(m_{1}, m_{2}\right)$ are given by

$$
\begin{aligned}
& C_{1}\left(m_{1}, m_{2}\right)=\mathbb{E}\left[S_{T, m_{1}} I_{\left\{S_{T, m_{1}} \geq K, V_{T, m_{2}} \geq D^{*}+S_{T, m_{1}}-K\right\}}\right], \\
& C_{2}\left(m_{1}, m_{2}\right)=-K \mathbb{E}\left[I_{\left\{S_{T, m_{1}} \geq K, V_{T, m_{2}} \geq D^{*}+S_{T, m_{1}}-K\right\}}\right], \\
& C_{3}\left(m_{1}, m_{2}\right)=\mathbb{E}\left[\frac{1-\alpha}{D^{*}+S_{T, m_{1}}-K} S_{T, m_{1}} V_{T, m_{2}} I_{\left\{S_{T, m_{1}} \geq K, V_{T, m_{2}}<D^{*}+S_{T, m_{1}}-K\right\}}\right], \\
& C_{4}\left(m_{1}, m_{2}\right)=-K \mathbb{E}\left[\frac{1-\alpha}{D^{*}+S_{T, m_{1}}-K} V_{T, m_{2}} I_{\left\{S_{T, m_{1}} \geq K, V_{T, m_{2}}<D^{*}+S_{T, m_{1}}-K\right\}}\right] .
\end{aligned}
$$

By complicated calculation, we can get the closed form of $C_{1}\left(m_{1}, m_{2}\right), C_{2}\left(m_{1}, m_{2}\right)$, $C_{3}\left(m_{1}, m_{2}\right)$, and $C_{4}\left(m_{1}, m_{2}\right)$ respectively:

$$
\begin{aligned}
& C_{1}\left(m_{1}, m_{2}\right)= S_{0} \exp \left\{\left(r-k_{S} \lambda_{S}^{*}\right) T+m_{1} \mu_{1}+\frac{1}{2} m_{1} \sigma_{1}^{2}\right\} N_{2}\left(a_{1}\left(m_{1}\right), a_{2}\left(m_{1}, m_{2}\right), \delta\right), \\
& C_{2}\left(m_{1}, m_{2}\right)=-K N_{2}\left(b_{1}\left(m_{1}\right), b_{2}\left(m_{1}, m_{2}\right), \delta\right), \\
& C_{3}\left(m_{1}, m_{2}\right)= H \exp \left\{\frac{\left(g+U\left(m_{1}\right)+m V\left(m_{2}\right)\right)^{2}}{2}\right. \\
&\left.+\frac{2 \delta\left(g+U\left(m_{1}\right)+m V\left(m_{2}\right)\right) \eta V\left(m_{2}\right)+\left(\eta V\left(m_{2}\right)\right)^{2}}{2}\right\} \\
& \cdot N_{2}\left(c_{1}\left(m_{1}, m_{2}\right), c_{2}\left(m_{1}, m_{2}\right),-\delta\right), \\
& C_{4}\left(m_{1}, m_{2}\right) \quad \\
&=-\tilde{H} \exp \left\{\frac{\left(g+m V\left(m_{2}\right)\right)^{2}+2 \delta\left(g+m V\left(m_{2}\right)\right) \eta V\left(m_{2}\right)+\left(\eta V\left(m_{2}\right)\right)^{2}}{2}\right\} \\
& \cdot N_{2}\left(d_{1}\left(m_{1}, m_{2}\right), d_{2}\left(m_{1}, m_{2}\right),-\delta\right),
\end{aligned}
$$

where $N_{2}(\cdot, \cdot, \cdot)$ denotes the two-dimensional normal distribution function. The calculation process can be found in the Appendix, and the above parameter values are given as follows:

$$
\begin{aligned}
& a_{1}\left(m_{1}\right)=\frac{\ln \frac{S_{0}}{K}+\left(r+\frac{1}{2} \sigma_{S}^{2}-k_{S} \lambda_{S}^{*}\right) T+m_{1} \mu_{1}+m_{1} \sigma_{1}^{2}}{\sqrt{\sigma_{S}^{2} T+m_{1} \sigma_{1}^{2}}}, \\
& a_{2}\left(m_{1}, m_{2}\right)=\frac{-b+m p+(\bar{\rho}-m) \sqrt{\sigma_{S}^{2} T+m_{1} \sigma_{1}^{2}}}{\sqrt{1-2 \bar{\rho} m+m^{2}}},
\end{aligned}
$$




$$
\begin{aligned}
& b_{1}\left(m_{1}\right)=\frac{\ln \frac{S_{0}}{K}+\left(r-\frac{1}{2} \sigma_{S}^{2}-k_{S} \lambda_{S}^{*}\right) T+m_{1} \mu_{1}}{\sqrt{\sigma_{S}^{2} T+m_{1} \sigma_{1}^{2}}}, \\
& b_{2}\left(m_{1}, m_{2}\right)=-\frac{b-m p}{\sqrt{1-2 \bar{\rho} m+m^{2}}}, \\
& c_{1}\left(m_{1}, m_{2}\right)=b_{1}\left(m_{1}\right)+\left(g+\sqrt{\sigma_{S}^{2} T+m_{1} \sigma_{1}^{2}}+m \sqrt{\sigma_{V}^{2} T+m_{2} \sigma_{2}^{2}}\right) \\
& +\delta \sqrt{1-2 \bar{\rho} m+m^{2}} \sqrt{\sigma_{V}^{2} T+m_{2} \sigma_{2}^{2}} \\
& c_{2}\left(m_{1}, m_{2}\right)=-b_{2}\left(m_{1}, m_{2}\right)-\delta\left(g+\sqrt{\sigma_{S}^{2} T+m_{1} \sigma_{1}^{2}}+m \sqrt{\sigma_{V}^{2} T+m_{2} \sigma_{2}^{2}}\right) \\
& -\sqrt{1-2 \bar{\rho} m+m^{2}} \sqrt{\sigma_{V}^{2} T+m_{2} \sigma_{2}^{2}} \\
& d_{1}\left(m_{1}, m_{2}\right)=b_{1}\left(m_{1}\right)+\left(g+m \sqrt{\sigma_{V}^{2} T+m_{2} \sigma_{2}^{2}}\right)+\delta \sqrt{1-2 \bar{\rho} m+m^{2}} \sqrt{\sigma_{V}^{2} T+m_{2} \sigma_{2}^{2}}, \\
& d_{2}\left(m_{1}, m_{2}\right)=-b_{2}\left(m_{1}, m_{2}\right)-\delta\left(g+m \sqrt{\sigma_{V}^{2} T+m_{2} \sigma_{2}^{2}}\right) \\
& -\sqrt{1-2 \bar{\rho} m+m^{2}} \sqrt{\sigma_{V}^{2} T+m_{2} \sigma_{2}^{2}} \\
& \bar{\rho}\left(m_{1}, m_{2}\right)=\frac{\sigma_{S} \sigma_{V} T}{\sqrt{\sigma_{S}^{2} T+m_{1} \sigma_{1}^{2}} \sqrt{\sigma_{V}^{2} T+m_{2} \sigma_{2}^{2}}} \rho=\bar{\rho}, \\
& b=\frac{\ln \left(\frac{D^{*}-K+S_{0} \exp \left\{M_{1}\left(m_{1}\right)+\sqrt{\sigma_{S}^{2} T+m_{1} \sigma_{1}^{2}} \cdot p\right\}}{V_{0}}\right)-M_{2}\left(m_{2}\right)}{\sqrt{\sigma_{V}^{2} T+m_{2} \sigma_{2}^{2}}} \\
& m=\frac{\sqrt{\sigma_{S}^{2} T+m_{1} \sigma_{1}^{2}}}{\sqrt{\sigma_{V}^{2} T+m_{2} \sigma_{2}^{2}}} \\
& \frac{S_{0} \exp \left\{\left(r-\frac{1}{2} \sigma_{S}^{2}-k_{S} \lambda_{S}^{*}\right) T+m_{1} \mu_{1}+\sqrt{\sigma_{S}^{2} T+m_{1} \sigma_{1}^{2}} p\right\}}{D^{*}-K+S_{0} \exp \left\{\left(r-\frac{1}{2} \sigma_{S}^{2}-k_{S} \lambda_{S}^{*}\right) T+m_{1} \mu_{1}+\sqrt{\sigma_{S}^{2} T+m_{1} \sigma_{1}^{2}} p\right\}}, \\
& \delta=\frac{\bar{\rho}-m}{\sqrt{1-2 \bar{\rho} m+m^{2}}} \\
& g=\frac{-S_{0} \sqrt{\sigma_{S}^{2} T+m_{1} \sigma_{1}^{2}} \exp \left\{M_{1}\left(m_{1}\right)+\sqrt{\sigma_{S}^{2} T+m_{1} \sigma_{1}^{2}} q\right\}}{D^{*}-K+S_{0} \exp \left\{M_{1}\left(m_{1}\right)+\sqrt{\sigma_{S}^{2} T+m_{1} \sigma_{1}^{2}} q\right\}}, \\
& H=\frac{(1-\alpha) S_{0} V_{0} \exp \left(M_{1}\left(m_{1}\right)+M_{2}\left(m_{2}\right)\right) \exp (-g q)}{D^{*}-K+S_{0} \exp \left(M_{1}\left(m_{1}\right)+\sqrt{\sigma_{S}^{2} T+m_{1} \sigma_{1}^{2}} q\right)}, \\
& \tilde{H}=\frac{(1-\alpha) K V_{0} \exp \left(M_{2}\left(m_{2}\right)\right) \exp (-g q)}{D^{*}-K+S_{0} \exp \left(M_{1}\left(m_{1}\right)+\sqrt{\sigma_{S}^{2} T+m_{1} \sigma_{1}^{2}} q\right)}, \\
& \eta=\sqrt{1-2 \bar{\rho} m+m^{2}}, \\
& U\left(m_{1}\right)=\sqrt{\sigma_{S}^{2} T+m_{1} \sigma_{1}^{2}}, \\
& V\left(m_{2}\right)=\sqrt{\sigma_{V}^{2} T+m_{2} \sigma_{2}^{2}},
\end{aligned}
$$


where $p$ and $q$ are two design parameters, around which we take the first Taylor expansions in the Appendix. From Equation (2.5) we can derive that

$$
\begin{aligned}
C_{m_{1}, m_{2}} & =S_{0} \exp \left\{-k_{S} \lambda_{S}^{*} T+m_{1} \mu_{1}+\frac{1}{2} m_{1} \sigma_{1}^{2}\right\} N_{2}\left(a_{1}\left(m_{1}\right), a_{2}\left(m_{1}, m_{2}\right), \delta\right) \\
& -K \mathrm{e}^{-r T} N_{2}\left(b_{1}\left(m_{1}\right), b_{2}\left(m_{1}, m_{2}\right), \delta\right) \\
& +H \mathrm{e}^{-r T} \exp \left\{\frac{\left(g+U\left(m_{1}\right)+m V\left(m_{2}\right)\right)^{2}}{2}\right. \\
& \left.+\frac{2 \delta\left(g+U\left(m_{1}\right)+m V\left(m_{2}\right)\right) \eta V\left(m_{2}\right)+\left(\eta V\left(m_{2}\right)\right)^{2}}{2}\right\} \\
& \cdot N_{2}\left(c_{1}\left(m_{1}, m_{2}\right), c_{2}\left(m_{1}, m_{2}\right),-\delta\right) \\
& -\tilde{H} \mathrm{e}^{-r T} \exp \left\{\frac{\left(g+m V\left(m_{2}\right)\right)^{2}+2 \delta\left(g+m V\left(m_{2}\right)\right) \eta V\left(m_{2}\right)+\left(\eta V\left(m_{2}\right)\right)^{2}}{2}\right\} \\
& \cdot N_{2}\left(d_{1}\left(m_{1}, m_{2}\right), d_{2}\left(m_{1}, m_{2}\right),-\delta\right) .
\end{aligned}
$$

Thus, we can obtain the analytical pricing formula of the vulnerable European call options

$$
\begin{aligned}
C^{*}= & \sum_{n=0}^{\infty} \sum_{m_{1}=n m_{2}=n}^{\infty} \frac{(\lambda T)^{n}}{n !} \frac{\left(\lambda_{S} T\right)^{m_{1}-n}}{\left(m_{1}-n\right) !} \frac{\left(\lambda_{V} T\right)^{m_{2}-n}}{\left(m_{2}-n\right) !} \mathrm{e}^{-\lambda T-\lambda_{S} T-\lambda_{V} T} \\
& \cdot\left\{S_{0} \exp \left\{-k_{S} \lambda_{S}^{*} T+m_{1} \mu_{1}+\frac{1}{2} m_{1} \sigma_{1}^{2}\right\} N_{2}\left(a_{1}\left(m_{1}\right), a_{2}\left(m_{1}, m_{2}\right), \delta\right)\right. \\
& -K \mathrm{e}^{-r T} N_{2}\left(b_{1}\left(m_{1}\right), b_{2}\left(m_{1}, m_{2}\right), \delta\right) \\
& +H \mathrm{e}^{-r T} \exp \left\{\frac{\left(g+U\left(m_{1}\right)+m V\left(m_{2}\right)\right)^{2}}{2}\right. \\
& \left.+\frac{2 \delta\left(g+U\left(m_{1}\right)+m V\left(m_{2}\right)\right) \eta V\left(m_{2}\right)+\left(\eta V\left(m_{2}\right)\right)^{2}}{2}\right\} \\
& \cdot N_{2}\left(c_{1}\left(m_{1}, m_{2}\right), c_{2}\left(m_{1}, m_{2}\right),-\delta\right) \\
& -\tilde{H} \mathrm{e}^{-r T} \exp \left\{\frac{\left(g+m V\left(m_{2}\right)\right)^{2}+2 \delta\left(g+m V\left(m_{2}\right)\right) \eta V\left(m_{2}\right)+\left(\eta V\left(m_{2}\right)\right)^{2}}{2}\right\} \\
& \left.\cdot N_{2}\left(d_{1}\left(m_{1}, m_{2}\right), d_{2}\left(m_{1}, m_{2}\right),-\delta\right)\right\} .
\end{aligned}
$$

Similarly, the explicit form of vulnerable European put option is expressed as

$$
\begin{aligned}
P^{*}= & \sum_{n=0}^{\infty} \sum_{m_{1}=n m_{2}=n}^{\infty} \sum^{\infty} \frac{(\lambda T)^{n}}{n !} \frac{\left(\lambda_{S} T\right)^{m_{1}-n}}{\left(m_{1}-n\right) !} \frac{\left(\lambda_{V} T\right)^{m_{2}-n}}{\left(m_{2}-n\right) !} \mathrm{e}^{-\lambda T-\lambda_{S} T-\lambda_{V} T} \\
& \cdot\left\{-S_{0} \exp \left\{-k_{S} \lambda_{S}^{*} T+m_{1} \mu_{1}+\frac{1}{2} m_{1} \sigma_{1}^{2}\right\} N_{2}\left(-a_{1}\left(m_{1}\right), a_{2}\left(m_{1}, m_{2}\right),-\delta\right)\right. \\
& +K \mathrm{e}^{-r T} N_{2}\left(-b_{1}\left(m_{1}\right), b_{2}\left(m_{1}, m_{2}\right),-\delta\right)
\end{aligned}
$$




$$
\begin{aligned}
& -H \mathrm{e}^{-r T} \exp \left\{\frac{\left(g+U\left(m_{1}\right)+m V\left(m_{2}\right)\right)^{2}}{2}\right. \\
& \left.+\frac{2 \delta\left(g+U\left(m_{1}\right)+m V\left(m_{2}\right)\right) \eta V\left(m_{2}\right)+\left(\eta V\left(m_{2}\right)\right)^{2}}{2}\right\} \\
& \cdot N_{2}\left(-c_{1}\left(m_{1}, m_{2}\right), c_{2}\left(m_{1}, m_{2}\right), \delta\right) \\
& +\tilde{H} \mathrm{e}^{-r T} \exp \left\{\frac{\left(g+m V\left(m_{2}\right)\right)^{2}+2 \delta\left(g+m V\left(m_{2}\right)\right) \eta V\left(m_{2}\right)+\left(\eta V\left(m_{2}\right)\right)^{2}}{2}\right\} \\
& \left.\cdot N_{2}\left(-d_{1}\left(m_{1}, m_{2}\right), d_{2}\left(m_{1}, m_{2}\right), \delta\right)\right\} .
\end{aligned}
$$

\subsection{Three specific examples}

As our particular cases, we will give the following three examples: classical Black-Scholes model, Merton's jump-diffusion model, and the model in [10].

Example 1 (Classical Black-Scholes model) When the counterparty risk and jump risk do not exist, we have $D^{*}+S_{T}-K=0, \lambda=\lambda_{S}=\lambda_{V}=0$, and $n=n_{1}=n_{2}=0$. Then Equation (2.8) is the classical Black-Scholes equation. This time, we can get

$$
\begin{aligned}
& C_{1}(0,0)=\mathbb{E}\left[S_{T, 0} I_{\left\{S_{T, 0} \geq K\right\}}\right]=S_{0} \mathbb{E}\left[\frac{S_{T, 0}}{S_{0}} I_{\left\{\ln \frac{S_{T, 0}}{S_{0}} \geq \ln \frac{K}{S_{0}}\right\}}\right] \\
& =S_{0} \mathbb{E}[\frac{S_{T, 0}}{S_{0}} \underbrace{}_{\left\{\xi_{1} \geq \frac{\ln \frac{K}{S_{0}}-M_{1}(0)}{\sigma_{S} \sqrt{T}}\right\}}] \\
& =S_{0} \int_{-b_{1}(0)}^{\infty} \mathrm{e}^{M_{1}(0)+\sigma_{S} \sqrt{T} x} \frac{1}{\sqrt{2 \pi}} \mathrm{e}^{-\frac{x^{2}}{2}} d x \\
& =S_{0} \int_{-b_{1}(0)}^{\infty} \mathrm{e}^{r T-\frac{1}{2} \sigma_{S}^{2} T+\sigma_{S} \sqrt{T} x} \frac{1}{\sqrt{2 \pi}} \mathrm{e}^{-\frac{x^{2}}{2}} d x \\
& =S_{0} \mathrm{e}^{r T} \int_{-b_{1}(0)}^{\infty} \frac{1}{\sqrt{2 \pi}} \mathrm{e}^{-\frac{\left(x-\sigma_{S} \sqrt{T}\right)^{2}}{2}} d x \\
& =S_{0} \mathrm{e}^{r T} \int_{-b_{1}(0)-\sigma_{S} \sqrt{T}}^{\infty} \frac{1}{\sqrt{2 \pi}} \mathrm{e}^{-\frac{u^{2}}{2}} d u \\
& =S_{0} \mathrm{e}^{r T} N_{1}\left(A_{1}\right) \text {, } \\
& C_{2}(0,0)=-K \mathbb{E}\left[I_{\left\{S_{T, 0} \geq K\right\}}\right]=-K \mathbb{E}\left[I_{\left\{\ln \frac{S_{T, 0}}{S_{0}} \geq \ln \frac{K}{S_{0}}\right\}}\right] \\
& =-K \mathbb{E}\left[I \quad \ln \frac{\ln \frac{K}{S_{0}}-M_{1}(0)}{\sigma_{S} \sqrt{T}}\right\} \\
& =-K \int_{-b_{1}(0)}^{\infty} \frac{1}{\sqrt{2 \pi}} \mathrm{e}^{-\frac{u^{2}}{2}} d u \\
& =-K N_{1}\left(B_{1}\right) \text {, } \\
& C_{3}(0,0)=C_{4}(0,0)=0 \text {, }
\end{aligned}
$$

where $N_{1}(\cdot)$ denotes the standard normal distribution function. 
Moreover, $a_{1}(0)$ and $b_{1}(0)$ reduce to $A_{1}=\frac{\ln \frac{S_{0}}{K}+\left(r+\frac{1}{2} \sigma_{S}^{2}\right) T}{\sigma_{S} \sqrt{T}}$ and $B_{1}=A_{1}-\sigma_{S} \sqrt{T}$, respectively. Equation (2.8) can be rewritten as

$$
C^{*}=S_{0} N_{1}\left(A_{1}\right)-K \mathrm{e}^{-r T} N_{1}\left(B_{1}\right)
$$

Example 2 (Merton's jump-diffusion model) Since there is no risk of default, we have $D^{*}+$ $S_{T}-K=0$. Thus,

$$
\begin{aligned}
& C_{1}\left(m_{1}, m_{2}\right)=\mathbb{E}\left[S_{T, m_{1}} I_{\left\{S_{T, m_{1}} \geq K\right\}}\right]=S_{0} \mathbb{E}\left[\frac{S_{T, m_{1}}}{S_{0}} I_{\left\{\ln \frac{S_{T, m_{1}}}{S_{0}} \geq \ln \frac{K}{S_{0}}\right\}}\right] \\
& =S_{0} \mathbb{E}\left[\mathrm{e}^{M_{1}\left(m_{1}\right)+\sqrt{\sigma_{S}^{2} T+m_{1} \sigma_{1}^{2}} \xi_{1}} I_{\left\{\xi_{1} \geq \frac{\ln \frac{K}{S_{0}}-M_{1}\left(m_{1}\right)}{\sigma_{S} \sqrt{T}}\right\}}\right] \\
& =S_{0} \int_{-b_{1}\left(m_{1}\right)}^{\infty} \mathrm{e}^{M_{1}\left(m_{1}\right)+\sqrt{\sigma_{S}^{2} T+m_{1} \sigma_{1}^{2}} u} \frac{1}{\sqrt{2 \pi}} \mathrm{e}^{-\frac{u^{2}}{2}} d u \\
& =S_{0} \mathrm{e}^{r T-k_{S} \lambda_{S}^{*} T+m_{1} \mu_{1}+\frac{1}{2} m_{1} \sigma_{1}^{2}} \int_{-b_{1}\left(m_{1}\right)}^{\infty} \frac{1}{\sqrt{2 \pi}} \mathrm{e}^{-\frac{\left(u-\sqrt{\sigma_{S}^{2} T+m_{1} \sigma_{1}^{2}}\right)^{2}}{2}} d u \\
& =S_{0} \mathrm{e}^{r T-k_{S} \lambda_{S}^{*} T+m_{1} \mu_{1}+\frac{1}{2} m_{1} \sigma_{1}^{2}} \int_{-b_{1}\left(m_{1}\right)-\sqrt{\sigma_{S}^{2} T+m_{1} \sigma_{1}^{2}}}^{\infty} \frac{1}{\sqrt{2 \pi}} \mathrm{e}^{-\frac{x^{2}}{2}} d x \\
& =S_{0} \mathrm{e}^{r T-k_{S} \lambda_{S}^{*} T+m_{1} \mu_{1}+\frac{1}{2} m_{1} \sigma_{1}^{2}} \int_{-a_{1}\left(m_{1}\right)}^{\infty} \frac{1}{\sqrt{2 \pi}} \mathrm{e}^{-\frac{x^{2}}{2}} d x \\
& =S_{0} \mathrm{e}^{r T-k_{S} \lambda_{S}^{*} T+m_{1} \mu_{1}+\frac{1}{2} m_{1} \sigma_{1}^{2}} N_{1}\left(a_{1}\left(m_{1}\right)\right), \\
& C_{2}\left(m_{1}, m_{2}\right)=-K \mathbb{E}\left[I_{\left\{S_{T, m_{1}} \geq K\right\}}\right]=-K \mathbb{E}\left[I_{\left\{\ln \frac{\left.S_{T, m_{1}} \geq \ln \frac{K}{S_{0}}\right\}}{S_{0}}\right]}\right] \\
& =-K \mathbb{E}\left[I_{\left\{\xi_{1} \geq \frac{\ln \frac{K}{S_{0}}-M_{1}\left(m_{1}\right)}{\sigma_{S} \sqrt{T}}\right\}}\right] \\
& =-K \int_{-b_{1}\left(m_{1}\right)}^{\infty} \frac{1}{\sqrt{2 \pi}} \mathrm{e}^{-\frac{u^{2}}{2}} d u \\
& =-K N_{1}\left(b_{1}\left(m_{1}\right)\right) \text {, } \\
& C_{3}(0,0)=C_{4}(0,0)=0 .
\end{aligned}
$$

Thus

$$
C_{m_{1}, m_{2}}=S_{0} \mathrm{e}^{-k s \lambda_{S}^{*} T+m_{1} \mu_{1}+\frac{1}{2} m_{1} \sigma_{1}^{2}} N_{1}\left(a_{1}\left(m_{1}\right)\right)-K \mathrm{e}^{-r T} N_{1}\left(b_{1}\left(m_{1}\right)\right) .
$$

As $C_{m_{1}, m_{2}}$ is irrelevant to $m_{2}$,

$$
\begin{aligned}
C^{*} & =\sum_{n=0}^{\infty} \sum_{m_{1}=n}^{\infty} \sum_{m_{2}=n}^{\infty} \frac{(\lambda T)^{n}}{n !} \frac{\left(\lambda_{S} T\right)^{m_{1}-n}}{\left(m_{1}-n\right) !} \frac{\left(\lambda_{V} T\right)^{m_{2}-n}}{\left(m_{2}-n\right) !} \mathrm{e}^{-\lambda T-\lambda_{S} T-\lambda_{V} T} C_{m_{1}, m_{2}} \\
& =\sum_{n=0}^{\infty} \sum_{m_{1}=n}^{\infty} \frac{(\lambda T)^{n}}{n !} \frac{\left(\lambda_{S} T\right)^{m_{1}-n}}{\left(m_{1}-n\right) !} \mathrm{e}^{-\lambda T-\lambda_{S} T} C_{m_{1}, m_{2}}\left(\sum_{n_{2}=0}^{\infty} \frac{\left(\lambda_{V} T\right)^{n_{2}}}{n_{2} !} \mathrm{e}^{-\lambda_{V} T}\right)
\end{aligned}
$$




$$
\begin{aligned}
= & \sum_{n=0}^{\infty} \sum_{m_{1}=n}^{\infty} \mathrm{e}^{-\lambda T-\lambda_{S} T} \frac{(\lambda T)^{n}}{n !} \frac{\left(\lambda_{S} T\right)^{m_{1}-n}}{\left(m_{1}-n\right) !} \\
& \cdot\left(S_{0} \mathrm{e}^{-k \lambda_{S}^{*} T+m_{1} \mu_{1}+\frac{1}{2} m_{1} \sigma_{1}^{2}} N_{1}\left(a_{1}\left(m_{1}\right)\right)-K \mathrm{e}^{-r T} N_{1}\left(b_{1}\left(m_{1}\right)\right)\right),
\end{aligned}
$$

where $a_{1}\left(m_{1}\right)$ and $b_{1}\left(m_{1}\right)$ can be found in (2.6). Noting that $(a+b)^{m}=\sum_{i=0}^{m} C_{m}^{i} a^{i} b^{m-i}$, we can represent $C^{*}$ as follows:

$$
C^{*}=\sum_{i=0}^{\infty} \mathrm{e}^{-\lambda_{S}^{*} T} \frac{\left(\lambda_{S}^{*} T\right)^{i}}{i !}\left(S_{0} \mathrm{e}^{-k_{S} \lambda_{S}^{*} T+i \mu_{1}+\frac{1}{2} i \sigma_{1}^{2}} N_{1}\left(a_{1}(i)\right)-K \mathrm{e}^{-r T} N_{1}\left(b_{1}(i)\right)\right) .
$$

Example 3 (Vulnerable non-jump Black-Scholes model in [10]) To simplify the formula, we can assume that the two design parameters $p$ and $q$ are equal. If there is no jump, that is, $\lambda=\lambda_{S}=\lambda_{V}=0, m_{1}=m_{2}=0, C_{1}(0,0), C_{2}(0,0), C_{3}(0,0)$, and $C_{4}(0,0)$ can be restated as follows:

$$
\begin{aligned}
C_{1}(0,0)= & S_{0} \mathrm{e}^{r T} N_{2}\left(b_{1}(0)+\sigma_{S} \sqrt{T},-\frac{b-m p}{\sqrt{1-2 \rho m+m^{2}}}+\delta \sigma_{S} \sqrt{T}, \delta\right), \\
C_{2}(0,0)= & -K N_{2}\left(b_{1}(0),-\frac{b-m p}{\sqrt{1-2 \rho m+m^{2}}}, \delta\right), \\
C_{3}(0,0)= & \frac{(1-\alpha) S_{0} V_{0} \exp \left\{2 r T+(\rho-m) \sigma_{S} \sigma_{V} T+\left(-2 \rho m+m^{2}\right) \frac{\sigma_{V}^{2}}{2} T-g p\right\}}{D^{*}-K+S_{0} \exp \left(\left(r-\frac{1}{2} \sigma_{S}^{2}\right) T+\sigma_{S} \sqrt{T} p\right)} \\
& \times N_{2}\left(c_{1}(0,0), c_{2}(0,0),-\delta\right), \\
C_{4}(0,0)= & \frac{(1-\alpha) K V_{0} \exp \left\{r T+\left(-2 \rho m+m^{2}\right) \frac{\sigma_{V}^{2}}{2} T-g p\right\}}{D^{*}-K+S_{0} \exp \left(\left(r-\frac{1}{2} \sigma_{S}^{2}\right) T+\sigma_{S} \sqrt{T} p\right)} N_{2}\left(d_{1}(0,0), d_{2}(0,0),-\delta\right),
\end{aligned}
$$

where the parameters are given by

$$
\begin{aligned}
& b_{1}(0)=\frac{\ln \frac{S_{0}}{K}+\left(r-\frac{1}{2} \sigma_{S}^{2}\right) T}{\sigma_{S} \sqrt{T}}, \\
& c_{1}(0,0)=b_{1}(0)+\left(\sigma_{S}+(\rho-m) \sigma_{V}\right) \sqrt{T}, \\
& c_{2}(0,0)=\frac{b-m p}{\sqrt{1-2 \rho m+m^{2}}}-\left(\delta \sigma_{S}-\sqrt{1-2 \rho m+m^{2}} \sigma_{V}\right) \sqrt{T}, \\
& d_{1}(0,0)=b_{1}(0)+(\rho-m) \sigma_{V} \sqrt{T}, \\
& d_{2}(0,0)=\frac{b-m p}{\sqrt{1-2 \rho m+m^{2}}}-\sqrt{1-2 \rho m+m^{2}} \sigma_{V} \sqrt{T}, \\
& \delta=\frac{\rho-m}{\sqrt{1-2 \rho m+m^{2}}}, \\
& b=\ln \left(\frac{D^{*}-K+S_{0} \exp \left(r-\frac{\sigma_{S}^{2}}{2} T+\sigma_{S} \sqrt{T} p\right)}{V_{0}}\right), \\
& m=\frac{\sigma_{S}}{\sigma_{V}}\left(\frac{S_{0} \exp \left(r-\frac{\sigma_{S}^{2}}{2} T+\sigma_{S} \sqrt{T} p\right)}{D^{*}-K+S_{0} \exp \left(r-\frac{\sigma_{S}^{2}}{2} T+\sigma_{S} \sqrt{T} p\right)}\right) .
\end{aligned}
$$


Equation (2.8) can be rewritten as

$$
\begin{aligned}
C^{*}= & S_{0} N_{2}\left(b_{1}(0)+\sigma_{S} \sqrt{T},-\frac{b-m p}{\sqrt{1-2 \rho m+m^{2}}}+\delta \sigma_{S} \sqrt{T}, \delta\right) \\
& -K \mathrm{e}^{-r T} N_{2}\left(b_{1}(0),-\frac{b-m p}{\sqrt{1-2 \rho m+m^{2}}}, \delta\right) \\
& +\frac{(1-\alpha) S_{0} V_{0} \exp \left\{r T+(\rho-m) \sigma_{S} \sigma_{V} T+\left(-2 \rho m+m^{2}\right) \frac{\sigma_{V}^{2}}{2} T-g p\right\}}{D^{*}-K+S_{0} \exp \left(\left(r-\frac{1}{2} \sigma_{S}^{2}\right) T+\sigma_{S} \sqrt{T} p\right)} \\
& \times N_{2}\left(c_{1}(0,0), c_{2}(0,0),-\delta\right) \\
& +\frac{(1-\alpha) K V_{0} \exp \left\{\left(-2 \rho m+m^{2}\right) \frac{\sigma_{V}^{2}}{2} T-g p\right\}}{D^{*}-K+S_{0} \exp \left(\left(r-\frac{1}{2} \sigma_{S}^{2}\right) T+\sigma_{S} \sqrt{T} p\right)} N_{2}\left(d_{1}(0,0), d_{2}(0,0),-\delta\right) .
\end{aligned}
$$

\section{Appendix}

In this section, we will give the detailed calculation needed to obtain the pricing formula.

Suppose that $\xi_{1}$ and $\xi_{2}$ both distribute normally $N_{1}(0,1)$ with correlation coefficient

$$
\bar{\rho}\left(m_{1}, m_{2}\right)=\frac{\sigma_{S} \sigma_{V} T}{\sqrt{\sigma_{S}^{2} T+m_{1} \sigma_{1}^{2}} \sqrt{\sigma_{V}^{2} T+m_{2} \sigma_{2}^{2}}} \rho .
$$

For simplicity, we sometimes denote it by $\bar{\rho}$. As we know, $\left(\ln \frac{S_{T, m_{1}}}{S_{0}}, \ln \frac{V_{T, m_{2}}}{V_{0}}\right)$ is bivariate normally distributed. Then the following equalities hold:

$$
\begin{aligned}
& \ln \frac{S_{T, m_{1}}}{S_{0}}=\left(r-\frac{1}{2} \sigma_{S}^{2}-k_{S} \lambda_{S}^{*}\right) T+m_{1} \mu_{1}+\sqrt{\sigma_{S}^{2} T+m_{1} \sigma_{1}^{2}} \xi_{1}, \\
& \ln \frac{V_{T, m_{2}}}{V_{0}}=\left(r-\frac{1}{2} \sigma_{V}^{2}-k_{V} \lambda_{V}^{*}\right) T+m_{2} \mu_{2}+\sqrt{\sigma_{V}^{2} T+m_{2} \sigma_{2}^{2}} \xi_{2} .
\end{aligned}
$$

Recall that

$$
\begin{aligned}
& M_{1}\left(m_{1}\right)=\mathbb{E}\left[\ln \frac{S_{T}, m_{1}}{S_{0}}\right]=\left(r-\frac{1}{2} \sigma_{S}^{2}-k_{S} \lambda_{S}^{*}\right) T+m_{1} \mu_{1}, \\
& M_{2}\left(m_{2}\right)=\mathbb{E}\left[\ln \frac{V_{T}, m_{2}}{V_{0}}\right]=\left(r-\frac{1}{2} \sigma_{V}^{2}-k_{V} \lambda_{V}^{*}\right) T+m_{2} \mu_{2} .
\end{aligned}
$$

Then $C_{1}\left(m_{1}, m_{2}\right)$ in Equation (2.5) is given by

$$
\begin{aligned}
& C_{1}\left(m_{1}, m_{2}\right) \\
& =\mathbb{E}\left[S_{T, m_{1}} I_{\left\{S_{T, m_{1}} \geq K, V_{T, m_{2}} \geq D^{*}-K+S_{T, m_{1}}\right\}}\right] \\
& =S_{0} \mathbb{E}\left[\frac{S_{T, m_{1}}}{S_{0}} I\left\{\ln \left(\frac{S_{T, m_{1}}}{S_{0}}\right) \geq \ln \frac{K}{S_{0}}, \ln \left(\frac{V_{T, m_{2}}}{V_{0}}\right) \geq \ln \left(\frac{D^{*}-K+S_{T, m_{1}}}{V_{0}}\right)\right\}\right] \\
& =S_{0} \mathbb{E}\left[\frac{S_{T, m_{1}}}{S_{0}} I\left\{\frac{\ln \left(\frac{S_{T, m_{1}}}{S_{0}}\right)-M_{1}\left(m_{1}\right)}{\sqrt{\sigma_{S}^{2} T+m_{1} \sigma_{1}^{2}}} \geq \frac{\ln \frac{K}{S_{0}}-M_{1}\left(m_{1}\right)}{\sqrt{\sigma_{S}^{2} T+m_{1} \sigma_{1}^{2}}}, \frac{\ln \left(\frac{V_{T, m_{2}}}{V_{0}}\right)-M_{2}\left(m_{2}\right)}{\sqrt{\sigma_{V}^{2} T+m_{2} \sigma_{2}^{2}}} \geq \frac{\ln \left(\frac{D^{*}-K+S_{T, m_{1}}}{V_{0}}\right)-M_{2}\left(m_{2}\right)}{\sqrt{\sigma_{V}^{2} T+m_{2} \sigma_{2}^{2}}}\right\}\right]
\end{aligned}
$$




$$
\begin{aligned}
& \left.=S_{0} \mathbb{E}\left[\mathrm{e}^{M_{1}\left(m_{1}\right)+\sqrt{\sigma_{S}^{2} T+m_{1} \sigma_{1}^{2}} \xi_{1}} I \quad \frac{\ln K}{S_{0}-M_{1}\left(m_{1}\right)}\right), \xi_{2} \geq \frac{\ln \left(\frac{\left.D^{*}-K+S_{T, m_{1}}\right)-M_{2}\left(m_{2}\right)}{\sqrt{\sigma_{S}^{2} T+m_{1} \sigma_{1}}}\right]}{\sqrt{\sigma_{V}^{2} T+m_{2} \sigma_{2}^{2}}}\right\} \\
& =S_{0} \int_{-b_{1}\left(m_{1}\right)}^{\infty} \int_{f(\widetilde{u})}^{\infty} \mathrm{e}^{M_{1}\left(m_{1}\right)+\sqrt{\sigma_{S}^{2} T+m_{1} \sigma_{1}^{2}} \widetilde{u}} \frac{1}{2 \pi \sqrt{1-\bar{\rho}\left(m_{1}, m_{2}\right)^{2}}} \\
& \cdot \exp \left\{-\frac{1}{2\left(1-\bar{\rho}\left(m_{1}, m_{2}\right)^{2}\right)}\left[\widetilde{u}^{2}-2 \bar{\rho}\left(m_{1}, m_{2}\right) \widetilde{u} \widetilde{v}+\widetilde{v}^{2}\right]\right\} d \widetilde{v} d \widetilde{u},
\end{aligned}
$$

where

$$
\begin{aligned}
& b_{1}\left(m_{1}\right)=\frac{\ln \frac{S_{0}}{K}+\left(r-\frac{1}{2} \sigma_{S}^{2}-k_{S} \lambda_{S}^{*}\right) T+m_{1} \mu_{1}}{\sqrt{\sigma_{S}^{2} T+m_{1} \sigma_{1}^{2}}}, \\
& f(\widetilde{u})=\frac{\ln \left(\frac{D^{*}-K+S_{0} \exp \left\{M_{1}\left(m_{1}\right)+\sqrt{\sigma_{S}^{2} T+m_{1} \sigma_{1}^{2}} \tilde{u}\right\}}{V_{0}}\right)-M_{2}\left(m_{2}\right)}{\sqrt{\sigma_{V}^{2} T+m_{2} \sigma_{2}^{2}}} .
\end{aligned}
$$

As in [10], we linearize the non-linear boundary $f(\widetilde{u})$ in the integral by taking a first order Taylor series expansion around the point " $p$ " as follows:

$$
f(\widetilde{u}) \approx f(p)+f^{\prime}(p)(\widetilde{u}-p)=b+m(\widetilde{u}-p),
$$

where

$$
b=f(p)=\frac{\ln \left(\frac{D^{*}-K+S_{0} \exp \left\{M_{1}\left(m_{1}\right)+\sqrt{\sigma_{S}^{2} T+m_{1} \sigma_{1}^{2}} p\right\}}{V_{0}}\right)-M_{2}\left(m_{2}\right)}{\sqrt{\sigma_{V}^{2} T+m_{2} \sigma_{2}^{2}}}
$$

and

$$
m=f^{\prime}(p)=\frac{\sqrt{\sigma_{S}^{2} T+m_{1} \sigma_{1}^{2}}}{\sqrt{\sigma_{V}^{2} T+m_{2} \sigma_{2}^{2}}} \cdot \frac{S_{0} \exp \left\{M_{1}\left(m_{1}\right)+\sqrt{\sigma_{S}^{2} T+m_{1} \sigma_{1}^{2}} p\right\}}{D^{*}-K+S_{0} \exp \left\{M_{1}\left(m_{1}\right)+\sqrt{\sigma_{S}^{2} T+m_{1} \sigma_{1}^{2}} p\right\}} .
$$

Now we derive

$$
\begin{aligned}
& C_{1}\left(m_{1}, m_{2}\right) \\
& =S_{0} \mathrm{e}^{M_{1}\left(m_{1}\right)} \int_{-b_{1}\left(m_{1}\right)}^{\infty} \int_{b+m(\widetilde{u}-p)}^{\infty} \mathrm{e}^{\sqrt{\sigma_{S}^{2} T+m_{1} \sigma_{1}^{2}} \widetilde{u}} \frac{1}{2 \pi \sqrt{1-\bar{\rho}\left(m_{1}, m_{2}\right)^{2}}} \\
& \quad \cdot \exp \left\{-\frac{1}{2\left(1-\bar{\rho}\left(m_{1}, m_{2}\right)^{2}\right)}\left[\widetilde{u}^{2}-2 \bar{\rho}\left(m_{1}, m_{2}\right) \widetilde{u} \widetilde{v}+\widetilde{v}^{2}\right]\right\} d \widetilde{v} d \widetilde{u} .
\end{aligned}
$$

Next we need to rotate the default boundary to eliminate its dependence on the variable $\widetilde{u}$. Consider the following transformation:

$$
\begin{aligned}
& \tilde{u}=\frac{1}{\sqrt{1+m^{2}}} \tilde{x}, \\
& \widetilde{v}=\tilde{y}+\frac{m}{\sqrt{1+m^{2}}} \tilde{x} .
\end{aligned}
$$


The determination of the Jacobian of this mapping is $|J|=\frac{1}{\sqrt{1+m^{2}}}$. Applying this transformation to $C_{1}\left(m_{1}, m_{2}\right)$ gives

$$
\begin{aligned}
C_{1}\left(m_{1}, m_{2}\right)= & S_{0} \mathrm{e}^{M_{1}\left(m_{1}\right)} \int_{-b_{1}\left(m_{1}\right) \sqrt{1+m^{2}}}^{\infty} \int_{b-m p}^{\infty} \frac{\exp \left(\frac{\sqrt{\sigma_{S}^{2} T+m_{1} \sigma_{1}^{2}}}{\sqrt{1+m^{2}}} \widetilde{x}\right)}{2 \pi \sqrt{1-\bar{\rho}^{2}}} \Omega(\widetilde{x}, \widetilde{y}) d \widetilde{y} d \widetilde{x} \\
= & S_{0} \mathrm{e}^{M_{1}\left(m_{1}\right)}\left(\frac{\sqrt{1-\delta^{2}}}{\sqrt{1-\bar{\rho}^{2}} \sqrt{1+m^{2}}}\right) \\
& \times \int_{-b_{1}\left(m_{1}\right) \sqrt{1+m^{2}}}^{\infty} \int_{b-m p}^{\infty} \frac{\exp \left(\frac{\sqrt{\sigma_{S}^{2} T+m_{1} \sigma_{1}^{2}}}{\sqrt{1+m^{2}}} \widetilde{x}\right)}{2 \pi \sqrt{1-\delta^{2}}} \Psi(\widetilde{x}, \widetilde{y}) d \tilde{y} d \widetilde{x},
\end{aligned}
$$

where

$$
\begin{aligned}
\Omega(\widetilde{x}, \widetilde{y})= & \exp \left\{-\frac{1}{2\left(1-\bar{\rho}^{2}\right)}\left[\left(\frac{\widetilde{x}}{\sqrt{1+m^{2}}}\right)^{2}-2 \bar{\rho}\left(\frac{\widetilde{x}}{\sqrt{1+m^{2}}}\right)\left(\tilde{y}+\frac{m}{\sqrt{1+m^{2}}} \widetilde{x}\right)\right.\right. \\
& \left.\left.+\left(\tilde{y}+\frac{m}{\sqrt{1+m^{2}}} \widetilde{x}\right)^{2}\right]\right\}, \\
\Psi(\widetilde{x}, \widetilde{y})= & \exp \left\{-\frac{1}{2\left(1-\delta^{2}\right)}\left[\left(\frac{\tilde{x}}{\sqrt{1+m^{2}}}\right)^{2}-2 \delta\left(\frac{\widetilde{x}}{\sqrt{1+m^{2}}}\right)\left(\frac{\tilde{y}}{\sqrt{1-2 \bar{\rho} m+m^{2}}}\right)\right.\right. \\
& \left.\left.+\left(\frac{\tilde{y}}{\sqrt{1-2 \bar{\rho} m+m^{2}}}\right)^{2}\right]\right\}
\end{aligned}
$$

and

$$
\delta=\frac{\bar{\rho}-m}{\sqrt{1-2 \bar{\rho} m+m^{2}}}
$$

Simplifying the exponential term $\Psi(\widetilde{x}, \widetilde{y})$, we obtain

$$
C_{1}\left(m_{1}, m_{2}\right)=S_{0} \mathrm{e}^{M_{1}\left(m_{1}\right)} \int_{-b_{1}\left(m_{1}\right)}^{\infty} \int_{\frac{b-m p}{\sqrt{1-2 \bar{\rho} m+m^{2}}}}^{\infty} \frac{\exp \left(\sqrt{\sigma_{S}^{2} T+m_{1} \sigma_{1}^{2}} \widetilde{q}\right)}{2 \pi \sqrt{1-\delta^{2}}} \Lambda(\widetilde{q}, \widetilde{r}) d \widetilde{r} d \widetilde{q},
$$

where

$$
\Lambda(\widetilde{q}, \widetilde{r})=\exp \left\{-\frac{1}{2\left(1-\delta^{2}\right)}\left[\widetilde{q}^{2}-2 \delta \widetilde{q} \widetilde{r}+\widetilde{r}^{2}\right]\right\}
$$

Now completing the square gives

$$
\begin{aligned}
C_{1}\left(m_{1}, m_{2}\right)= & S_{0} \mathrm{e}^{M_{1}\left(m_{1}\right)+\frac{\sigma_{S}^{2} T+m_{1} \sigma_{1}^{2}}{2}} \int_{-b_{1}\left(m_{1}\right)}^{\infty} \int_{\frac{b-m p}{\sqrt{1-2 \bar{\rho} m+m^{2}}}}^{\infty} \frac{1}{2 \pi \sqrt{1-\delta^{2}}} \\
& \cdot \exp \left\{-\frac{1}{2\left(1-\delta^{2}\right)}\left[\left(\widetilde{q}-\sqrt{\sigma_{S}^{2} T+m_{1} \sigma_{1}^{2}}\right)^{2}-2 \delta\left(\widetilde{q}-\sqrt{\sigma_{S}^{2} T+m_{1} \sigma_{1}^{2}}\right)\right.\right. \\
& \left.\left.\times\left(\widetilde{r}-\delta \sqrt{\sigma_{S}^{2} T+m_{1} \sigma_{1}^{2}}\right)+\left(\widetilde{r}-\delta \sqrt{\sigma_{S}^{2} T+m_{1} \sigma_{1}^{2}}\right)^{2}\right]\right\} d \widetilde{r} d \widetilde{q} .
\end{aligned}
$$


We denote

$$
\tilde{M}\left(m_{1}\right)=M_{1}\left(m_{1}\right)+\frac{\sigma_{S}^{2} T+m_{1} \sigma_{1}^{2}}{2}=\left(r-k_{S} \lambda_{S}^{*}\right) T+m_{1} \mu_{1}+\frac{1}{2} m_{1} \sigma_{1}^{2} .
$$

Using a simple change of variables results in

$$
\begin{aligned}
C_{1}\left(m_{1}, m_{2}\right)= & S_{0} \mathrm{e}^{\widetilde{M_{1}}\left(m_{1}\right)} \int_{-b_{1}\left(m_{1}\right)-\sqrt{\sigma_{S}^{2} T+m_{1} \sigma_{1}^{2}}}^{\infty} \int_{\frac{b-m p}{\sqrt{1-2 \bar{\rho} m+m^{2}}}-\delta \sqrt{\sigma_{S}^{2} T+m_{1} \sigma_{1}^{2}}}^{\infty} \frac{1}{2 \pi \sqrt{1-\delta^{2}}} \\
& \cdot \exp \left\{-\frac{1}{2\left(1-\delta^{2}\right)}\left[\widetilde{w}^{2}-2 \delta \widetilde{w} \widetilde{z}+\widetilde{z}^{2}\right]\right\} d \widetilde{z} d \widetilde{w} \\
= & S_{0} \mathrm{e}^{\widetilde{M_{1}}\left(m_{1}\right)} N_{2}\left(b_{1}\left(m_{1}\right)+\sqrt{\sigma_{S}^{2} T+m_{1} \sigma_{1}^{2}},\right. \\
& \left.-\frac{b-m p}{\sqrt{1-2 \bar{\rho} m+m^{2}}}+\delta \sqrt{\sigma_{S}^{2} T+m_{1} \sigma_{1}^{2}}, \delta\right),
\end{aligned}
$$

where $N_{2}(\cdot, \cdot, \cdot)$ denotes the two-dimensional normal distribution function.

In order to simplify the expression of parameters, we represent

$$
\begin{aligned}
& a_{1}\left(m_{1}\right)=b_{1}\left(m_{1}\right)+\sqrt{\sigma_{S}^{2} T+m_{1} \sigma_{1}^{2}}, \\
& a_{2}\left(m_{1}, m_{2}\right)=b_{2}\left(m_{1}, m_{2}\right)+\delta \sqrt{\sigma_{S}^{2} T+m_{1} \sigma_{1}^{2}}, \\
& b_{1}\left(m_{1}\right)=\frac{\ln \frac{S_{0}}{K}+\left(r-\frac{1}{2} \sigma_{S}^{2}-k_{S} \lambda_{S}^{*}\right) T+m_{1} \mu_{1}}{\sqrt{\sigma_{S}^{2} T+m_{1} \sigma_{1}^{2}}}, \\
& b_{2}\left(m_{1}, m_{2}\right)=-\frac{b-m p}{\sqrt{1-2 \bar{\rho} m+m^{2}}} .
\end{aligned}
$$

Thus,

$$
C_{1}\left(m_{1}, m_{2}\right)=S_{0} \mathrm{e}^{\widetilde{M_{1}}\left(m_{1}\right)} N_{2}\left(a_{1}\left(m_{1}\right), a_{2}\left(m_{1}, m_{2}\right), \delta\right) .
$$

For $C_{2}\left(m_{1}, m_{2}\right)$, the following expressions hold:

$$
\begin{aligned}
C_{2} & \left(m_{1}, m_{2}\right) \\
& =-K \mathbb{E}\left[I_{\left\{S_{T, m_{1}} \geq K, V_{T, m_{2}} \geq D^{*}+S_{T, m_{1}}-K\right\}}\right] \\
& =-\int_{-b_{1}\left(m_{1}\right)}^{\infty} \int_{f(\widetilde{u})}^{\infty} \frac{K}{2 \pi \sqrt{1-\bar{\rho}^{2}}} \exp \left\{-\frac{1}{2\left(1-\bar{\rho}^{2}\right)}\left[\widetilde{u}^{2}-2 \bar{\rho} \widetilde{u v}+\widetilde{v}^{2}\right]\right\} d \widetilde{v} d \widetilde{u} \\
& =-K \int_{-b_{1}\left(m_{1}\right)}^{\infty} \int_{b+m(\widetilde{u}-p)}^{\infty} \frac{1}{2 \pi \sqrt{1-\bar{\rho}^{2}}} \exp \left\{-\frac{1}{2\left(1-\bar{\rho}^{2}\right)}\left[\widetilde{u}^{2}-2 \bar{\rho} \widetilde{u v}+\widetilde{v}^{2}\right]\right\} d \widetilde{v} d \widetilde{u} \\
& =-K \int_{-b_{1}\left(m_{1}\right) \sqrt{1+m^{2}}}^{\infty} \int_{b-m p}^{\infty} \frac{1}{2 \pi \sqrt{1-\bar{\rho}^{2}}} \Omega(\widetilde{x}, \widetilde{y}) d \widetilde{y} d \widetilde{x} \\
& =-K\left(\frac{\sqrt{1-\delta^{2}}}{\sqrt{1-\bar{\rho}^{2}} \sqrt{1+m^{2}}}\right) \int_{-b_{1}\left(m_{1}\right) \sqrt{1+m^{2}}}^{\infty} \int_{b-m p}^{\infty} \frac{1}{2 \pi \sqrt{1-\delta^{2}}} \Psi(\widetilde{x}, \widetilde{y}) d \widetilde{y} d \widetilde{x}
\end{aligned}
$$




$$
\begin{aligned}
& =-K \int_{-b_{1}\left(m_{1}\right)}^{\infty} \int_{\frac{b-m p}{\sqrt{1-2 \bar{\rho} m+m^{2}}}}^{\infty} \frac{1}{2 \pi \sqrt{1-\delta^{2}}} \Lambda(\widetilde{q}, \widetilde{r}) d \widetilde{r} d \widetilde{q} \\
& =-K \int_{-b_{1}\left(m_{1}\right)}^{\infty} \int_{\frac{b-m p}{\sqrt{1-2 \bar{\rho} m+m^{2}}}}^{\infty} \frac{1}{2 \pi \sqrt{1-\delta^{2}}} \exp \left\{-\frac{1}{2\left(1-\delta^{2}\right)}\left[\widetilde{q}^{2}-2 \delta \widetilde{q} \widetilde{r}+\widetilde{r}^{2}\right]\right\} d \widetilde{r} d \widetilde{q} \\
& =-K N_{2}\left(b_{1}\left(m_{1}\right), b_{2}\left(m_{1}, m_{2}\right), \delta\right) .
\end{aligned}
$$

Recall that $\xi_{1}$ and $\xi_{2}$ both distribute normally $N_{1}(0,1)$ with correlation coefficient $\bar{\rho}$. Then $C_{3}\left(m_{1}, m_{2}\right)$ can be presented as follows:

$$
\begin{aligned}
& C_{3}\left(m_{1}, m_{2}\right) \\
&=\mathbb{E}\left[\frac{1-\alpha}{D^{*}+S_{T, m_{1}}-K} S_{T, m_{1}} V_{T, m_{2}} I_{\left\{S_{T, m_{1}} \geq K, V_{T, m_{2}}<D^{*}+S_{T, m_{1}}-K\right\}}\right] \\
&=\mathbb{E}\left[\frac{1-\alpha}{D^{*}-K+S_{0} \exp \left\{M_{1}\left(m_{1}\right)+\sqrt{\sigma_{S}^{2} T+m_{1} \sigma_{1}^{2}} \xi_{1}\right\}}\right. \\
& \quad \times S_{0} V_{0} \mathrm{e}^{M_{1}\left(m_{1}\right)+\sqrt{\sigma_{S}^{2} T+m_{1} \sigma_{1}^{2}} \xi_{1}} \mathrm{e}^{M_{2}\left(m_{2}\right)+\sqrt{\sigma_{V}^{2} T+m_{2} \sigma_{2}^{2}} \xi_{2}} \\
&\left.\cdot I_{\left\{\xi_{1} \geq-b_{1}\left(m_{1}\right), \xi_{2}<f(\tilde{u})\right\}}\right] \\
&=\int_{-b_{1}\left(m_{1}\right)}^{\infty} \int_{-\infty}^{f(\widetilde{u})}(1-\alpha) S_{0} V_{0} \\
& \quad \times \frac{\exp \left\{M_{1}\left(m_{1}\right)+M_{2}\left(m_{2}\right)+\sqrt{\sigma_{S}^{2} T+m_{1} \sigma_{1}^{2}} \widetilde{u}+\sqrt{\sigma_{V}^{2} T+m_{2} \sigma_{2}^{2}} \widetilde{v}\right\}}{D^{*}-K+S_{0} \exp \left\{M_{1}\left(m_{1}\right)+\sqrt{\sigma_{S}^{2} T+m_{1} \sigma_{1}^{2}} \widetilde{u}\right\}} \\
& \cdot \frac{1}{2 \pi \sqrt{1-\bar{\rho}^{2}}} \exp \left\{-\frac{1}{2\left(1-\bar{\rho}^{2}\right)}\left[\widetilde{u}^{2}-2 \tilde{\rho} \widetilde{u} \widetilde{v}+\widetilde{v}^{2}\right]\right\} d \widetilde{v} d \widetilde{u} .
\end{aligned}
$$

We also need to modify the denominator in the above integral. Let

$$
F(\widetilde{u})=\frac{1}{D^{*}-K+S_{0} \exp \left\{M_{1}\left(m_{1}\right)+\sqrt{\sigma_{S}^{2} T+m_{1} \sigma_{1}^{2}} \widetilde{u}\right\}} .
$$

Define $G(\widetilde{u})$ as follows:

$$
G(\widetilde{u})=\ln F(\widetilde{u})
$$

Now we can use other first order Taylor series expansions around the point " $q$ " to linearize $G(\widetilde{u})$ :

$$
G(\widetilde{u})=G(q)+G^{\prime}(q)(\widetilde{u}-q)=f+g(\widetilde{u}-q),
$$

where

$$
f=G(q)=\ln \left(\frac{1}{D^{*}-K+S_{0} \exp \left\{M_{1}\left(m_{1}\right)+\sqrt{\sigma_{S}^{2} T+m_{1} \sigma_{1}^{2}} q\right\}}\right)
$$


and

$$
g=G^{\prime}(q)=\frac{-S_{0} \sqrt{\sigma_{S}^{2} T+m_{1} \sigma_{1}^{2}} \exp \left\{M_{1}\left(m_{1}\right)+\sqrt{\sigma_{S}^{2} T+m_{1} \sigma_{1}^{2}} q\right\}}{D^{*}-K+S_{0} \exp \left\{M_{1}\left(m_{1}\right)+\sqrt{\sigma_{S}^{2} T+m_{1} \sigma_{1}^{2}} q\right\}} .
$$

Therefore

$$
\begin{aligned}
& F(\tilde{u}) \approx \exp [f+g(\tilde{u}-q)] \\
& F(\tilde{u}) \approx \frac{\exp \{g(\tilde{u}-q)\}}{D^{*}-K+S_{0} \exp \left\{M_{1}\left(m_{1}\right)+\sqrt{\sigma_{S}^{2} T+m_{1} \sigma_{1}^{2}} q\right\}}
\end{aligned}
$$

Now, substituting the Taylor series approximations into $C_{3}\left(m_{1}, m_{2}\right)$, we have

$$
\begin{aligned}
C_{3}\left(m_{1}, m_{2}\right)= & \frac{(1-\alpha) S_{0} V_{0} \exp \left(M_{1}\left(m_{1}\right)+M_{2}\left(m_{2}\right)\right) \exp (-g q)}{D^{*}-K+S_{0} \exp \left(M_{1}\left(m_{1}\right)+\sqrt{\left.\sigma_{S}^{2} T+m_{1} \sigma_{1}^{2} q\right)}\right.} \\
& \cdot \int_{-b_{1}\left(m_{1}\right)}^{\infty} \int_{-\infty}^{b+m(\widetilde{u}-p)} \exp \left\{\left(g+\sqrt{\sigma_{S}^{2} T+m_{1} \sigma_{1}^{2}}\right) \widetilde{u}+\sqrt{\sigma_{V}^{2} T+m_{2} \sigma_{2}^{2}} \widetilde{v}\right\} \\
& \cdot \frac{1}{2 \pi \sqrt{1-\bar{\rho}^{2}}} \exp \left\{-\frac{1}{2\left(1-\bar{\rho}^{2}\right)}\left[\widetilde{u}^{2}-2 \tilde{\rho} \widetilde{u} \tilde{v}+\widetilde{v}^{2}\right]\right\} d \widetilde{v} d \widetilde{u} .
\end{aligned}
$$

Denote

$$
H=\frac{(1-\alpha) S_{0} V_{0} \exp \left(M_{1}\left(m_{1}\right)+M_{2}\left(m_{2}\right)\right) \exp (-g q)}{D^{*}-K+S_{0} \exp \left(M_{1}\left(m_{1}\right)+\sqrt{\sigma_{S}^{2} T+m_{1} \sigma_{1}^{2}} q\right)},
$$

then we have

$$
\begin{aligned}
& C_{3}\left(m_{1}, m_{2}\right) \\
& =H \int_{-b_{1}\left(m_{1}\right)}^{\infty} \int_{-\infty}^{b+m(\widetilde{u}-p)} \frac{\exp \left\{\left(g+\sqrt{\sigma_{S}^{2} T+m_{1} \sigma_{1}^{2}}\right) \widetilde{u}+\sqrt{\sigma_{V}^{2} T+m_{2} \sigma_{2}^{2}} \widetilde{v}\right\}}{2 \pi \sqrt{1-\bar{\rho}^{2}}} \\
& \cdot \exp \left\{-\frac{1}{2\left(1-\bar{\rho}^{2}\right)}\left[\widetilde{u}^{2}-2 \bar{\rho} \widetilde{u} v+\widetilde{v}^{2}\right]\right\} d \widetilde{v} d \widetilde{u} \\
& =H \int_{-b_{1}\left(m_{1}\right) \sqrt{1+m^{2}}}^{\infty} \int_{-\infty}^{b-m p} \\
& \frac{\exp \left\{\left(\frac{g+\sqrt{\sigma_{S}^{2} T+m_{1} \sigma_{1}^{2}}+m \sqrt{\sigma_{V}^{2} T+m_{2} \sigma_{2}^{2}}}{\sqrt{1+m^{2}}}\right) \widetilde{x}+\sqrt{\sigma_{V}^{2} T+m_{2} \sigma_{2}^{2}} \widetilde{y}\right\}}{2 \pi \sqrt{1-\bar{\rho}^{2}}} \Omega(\widetilde{x}, \widetilde{y}) d \widetilde{y} d \widetilde{x} \\
& =H \frac{\sqrt{1-\delta^{2}}}{\sqrt{1-\bar{\rho}^{2}} \sqrt{1+m^{2}}} \int_{-b_{1}\left(m_{1}\right) \sqrt{1+m^{2}}}^{\infty} \int_{-\infty}^{b-m p} \\
& \frac{\exp \left\{\left(\frac{g+\sqrt{\sigma_{S}^{2} T+m_{1} \sigma_{1}^{2}}+m \sqrt{\sigma_{V}^{2} T+m_{2} \sigma_{2}^{2}}}{\sqrt{1+m^{2}}}\right) \widetilde{x}+\sqrt{\sigma_{V}^{2} T+m_{2} \sigma_{2}^{2}} \widetilde{y}\right\}}{2 \pi \sqrt{1-\delta^{2}}} \Psi(\widetilde{x}, \widetilde{y}) d \widetilde{y} d \widetilde{x} \\
& =H \int_{-b_{1}\left(m_{1}\right)}^{\infty} \int_{-\infty}^{\frac{b-m p}{\sqrt{1-2 \bar{\rho} m+m^{2}}}}
\end{aligned}
$$


Chou et al. Advances in Difference Equations

(2018) 2018:465

Page 18 of 21

$$
\begin{aligned}
& \frac{\exp \left\{\left(g+\sqrt{\sigma_{S}^{2} T+m_{1} \sigma_{1}^{2}}+m \sqrt{\sigma_{V}^{2} T+m_{2} \sigma_{2}^{2}}\right) \widetilde{q}+\sqrt{1-2 \bar{\rho} m+m^{2}} \sqrt{\sigma_{V}^{2} T+m_{2} \sigma_{2}^{2}} \widetilde{r}\right\}}{2 \pi \sqrt{1-\delta^{2}}} \\
& \times \Lambda(\widetilde{q}, \widetilde{r}) d \widetilde{r} d \widetilde{q} .
\end{aligned}
$$

Denote

$$
\begin{aligned}
& U\left(m_{1}\right)=\sqrt{\sigma_{S}^{2} T+m_{1} \sigma_{1}^{2}}, \\
& V\left(m_{2}\right)=\sqrt{\sigma_{V}^{2} T+m_{2} \sigma_{2}^{2}}, \\
& P=g+U\left(m_{1}\right)+m V\left(m_{2}\right), \\
& \tilde{P}=g+m V\left(m_{2}\right), \\
& \eta=\sqrt{1-2 \bar{\rho} m+m^{2}}, \\
& Q=\eta V\left(m_{2}\right),
\end{aligned}
$$

and

$$
\begin{aligned}
c_{1}\left(m_{1}, m_{2}\right)= & b_{1}\left(m_{1}\right)+\left(g+\sqrt{\sigma_{S}^{2} T+m_{1} \sigma_{1}^{2}}+m \sqrt{\sigma_{V}^{2} T+m_{2} \sigma_{2}^{2}}\right) \\
& +\delta \sqrt{1-2 \bar{\rho} m+m^{2}} \sqrt{\sigma_{V}^{2} T+m_{2} \sigma_{2}^{2}}, \\
c_{2}\left(m_{1}, m_{2}\right)= & -b_{2}\left(m_{1}, m_{2}\right)-\delta\left(g+\sqrt{\sigma_{S}^{2} T+m_{1} \sigma_{1}^{2}}+m \sqrt{\sigma_{V}^{2} T+m_{2} \sigma_{2}^{2}}\right) \\
& -\sqrt{1-2 \bar{\rho} m+m^{2}} \sqrt{\sigma_{V}^{2} T+m_{2} \sigma_{2}^{2}} .
\end{aligned}
$$

Now completing the square gives

$$
\begin{aligned}
& C_{3}\left(m_{1}, m_{2}\right) \\
&= H \exp \left\{\frac{\left(g+U\left(m_{1}\right)+m V\left(m_{2}\right)\right)^{2}}{2}\right. \\
&\left.+\frac{2 \delta\left(g+U\left(m_{1}\right)+m V\left(m_{2}\right)\right) \eta V\left(m_{2}\right)+\left(\eta V\left(m_{2}\right)\right)^{2}}{2}\right\} \\
& \cdot \int_{-b_{1}\left(m_{1}\right)}^{\infty} \int_{-\infty}^{\frac{b-m p}{\sqrt{1-2 \bar{\rho} m+m^{2}}}} \frac{1}{2 \pi \sqrt{1-\delta^{2}}} \\
& \times \exp \left\{-\frac{1}{2\left(1-\delta^{2}\right)}\left[(\widetilde{q}-P-\delta Q)^{2}-2 \delta(\widetilde{q}-P-\delta Q)\right.\right. \\
&\left.\left.+(\widetilde{r}-\delta P-Q)+(\widetilde{r}-\delta P-Q)^{2}\right]\right\} d \widetilde{r} d \widetilde{q} \\
&= H \exp \left\{\frac{\left(g+U\left(m_{1}\right)+m V\left(m_{2}\right)\right)^{2}}{2}\right. \\
&\left.+\frac{2 \delta\left(g+U\left(m_{1}\right)+m V\left(m_{2}\right)\right) \eta V\left(m_{2}\right)+\left(\eta V\left(m_{2}\right)\right)^{2}}{2}\right\} \\
& \cdot \int_{-b_{1}\left(m_{1}\right)-\left(g+U\left(m_{1}\right)+m V\left(m_{2}\right)\right)-\delta \eta V\left(m_{2}\right)}^{\infty} \int_{-\infty} \frac{b-m p}{\sqrt{1-2 \bar{\rho} m+m^{2}}}-\delta\left(g+U\left(m_{1}\right)+m V\left(m_{2}\right)\right)-\eta V\left(m_{2}\right)
\end{aligned}
$$




$$
\begin{aligned}
& \frac{1}{2 \pi \sqrt{1-\delta^{2}}} \exp \left\{-\frac{1}{2\left(1-\delta^{2}\right)}\left[\widetilde{w}^{2}-2 \delta \widetilde{w} \widetilde{z}+\widetilde{z}^{2}\right]\right\} d \widetilde{z} d \widetilde{w} \\
= & H \exp \left\{\frac{\left(g+U\left(m_{1}\right)+m V\left(m_{2}\right)\right)^{2}}{2}\right. \\
& \left.+\frac{2 \delta\left(g+U\left(m_{1}\right)+m V\left(m_{2}\right)\right) \eta V\left(m_{2}\right)+\left(\eta V\left(m_{2}\right)\right)^{2}}{2}\right\} \\
& \cdot N_{2}\left(c_{1}\left(m_{1}, m_{2}\right), c_{2}\left(m_{1}, m_{2}\right),-\delta\right) .
\end{aligned}
$$

Similarly, $C_{4}\left(m_{1}, m_{2}\right)$ can be written as

$$
\begin{aligned}
& C_{4}\left(m_{1}, m_{2}\right) \\
& =-K \mathbb{E}\left[\frac{1-\alpha}{D^{*}+S_{T, m_{1}}-K} V_{T, m_{2}} I_{\left\{S_{T, m_{1}} \geq K, V_{T, m_{2}}<D^{*}+S_{T, m_{1}}-K\right\}}\right] \\
& =-K \mathbb{E}\left[\frac{1-\alpha}{D^{*}-K+S_{0} \exp \left\{M_{1}\left(m_{1}\right)+\sqrt{\sigma_{S}^{2} T+m_{1} \sigma_{1}^{2}} \xi_{1}\right\}}\right. \\
& \left.\times V_{0} \mathrm{e}^{M_{2}\left(m_{2}\right)+\sqrt{\sigma_{V}^{2} T+m_{2} \sigma_{2}^{2}} \xi_{2}} I_{\left\{\xi_{1} \geq-b_{1}\left(m_{1}\right), \xi_{2}<f(\tilde{u})\right\}}\right] \\
& =-\int_{-b_{1}\left(m_{1}\right)}^{\infty} \int_{-\infty}^{f(\widetilde{u})} \frac{(1-\alpha) K V_{0} \exp \left\{M_{2}\left(m_{2}\right)+\sqrt{\sigma_{V}^{2} T+m_{2} \sigma_{2}^{2}} \widetilde{v}\right\}}{D^{*}-K+S_{0} \exp \left\{M_{1}\left(m_{1}\right)+\sqrt{\sigma_{S}^{2} T+m_{1} \sigma_{1}^{2}} \widetilde{u}\right\}} \\
& \cdot \frac{1}{2 \pi \sqrt{1-\bar{\rho}^{2}}} \exp \left\{-\frac{1}{2\left(1-\bar{\rho}^{2}\right)}\left[\widetilde{u}^{2}-2 \bar{\rho} \widetilde{u} \widetilde{v}+\widetilde{v}^{2}\right]\right\} d \widetilde{v} d \widetilde{u} \\
& =-\frac{(1-\alpha) K V_{0} \exp \left(M_{2}\left(m_{2}\right)\right) \exp (-g q)}{D^{*}-K+S_{0} \exp \left(M_{1}\left(m_{1}\right)+\sqrt{\sigma_{S}^{2} T+m_{1} \sigma_{1}^{2}} q\right)} \\
& \times \int_{-b_{1}\left(m_{1}\right)}^{\infty} \int_{-\infty}^{b+m(\widetilde{u}-p)} \exp \left\{g \widetilde{u}+\sqrt{\sigma_{V}^{2} T+m_{2} \sigma_{2}^{2}} \widetilde{v}\right\} \\
& \cdot \frac{1}{2 \pi \sqrt{1-\bar{\rho}^{2}}} \exp \left\{-\frac{1}{2\left(1-\bar{\rho}^{2}\right)}\left[\widetilde{u}^{2}-2 \bar{\rho} \widetilde{u} \widetilde{v}+\widetilde{v}^{2}\right]\right\} d \widetilde{v} d \widetilde{u} \text {. }
\end{aligned}
$$

Denote

$$
\tilde{H}=\frac{(1-\alpha) K V_{0} \exp \left(M_{2}\left(m_{2}\right)\right) \exp (-g q)}{D^{*}-K+S_{0} \exp \left(M_{1}\left(m_{1}\right)+\sqrt{\sigma_{S}^{2} T+m_{1} \sigma_{1}^{2}} q\right)},
$$

then we have

$$
\begin{aligned}
& C_{4}\left(m_{1}, m_{2}\right) \\
& =-\tilde{H} \int_{-b_{1}\left(m_{1}\right)}^{\infty} \int_{-\infty}^{b+m(\widetilde{u}-p)} \exp \left\{g \widetilde{u}+\sqrt{\sigma_{V}^{2} T+m_{2} \sigma_{2}^{2}} \widetilde{v}\right\} \\
& \quad \frac{1}{2 \pi \sqrt{1-\bar{\rho}^{2}}} \exp \left\{-\frac{1}{2\left(1-\bar{\rho}^{2}\right)}\left[\widetilde{u}^{2}-2 \bar{\rho} \widetilde{u} \widetilde{v}+\widetilde{v}^{2}\right]\right\} d \widetilde{v} d \widetilde{u}
\end{aligned}
$$




$$
\begin{aligned}
= & -\tilde{H} \int_{-b_{1}\left(m_{1}\right) \sqrt{1+m^{2}}}^{\infty} \int_{-\infty}^{b-m p} \frac{\exp \left\{\left(\frac{g+m \sqrt{\sigma_{V}^{2} T+m_{2} \sigma_{2}^{2}}}{\sqrt{1+m^{2}}}\right) \widetilde{x}+\sqrt{\sigma_{V}^{2} T+m_{2} \sigma_{2}^{2}} \widetilde{y}\right\}}{2 \pi \sqrt{1-\bar{\rho}^{2}}} \Omega(\widetilde{x}, \widetilde{y}) d \tilde{y} d \tilde{x} \\
= & -\tilde{H} \frac{\sqrt{1-\delta^{2}}}{\sqrt{1-\bar{\rho}^{2}} \sqrt{1+m^{2}}} \int_{-b_{1}\left(m_{1}\right) \sqrt{1+m^{2}}}^{\infty} \int_{-\infty}^{b-m p} \\
& \frac{\exp \left\{\frac{\left.\left(\frac{g+m \sqrt{\sigma_{V}^{2} T+m_{2} \sigma_{2}^{2}}}{\sqrt{1+m^{2}}}\right) \tilde{x}+\sqrt{\sigma_{V}^{2} T+m_{2} \sigma_{2}^{2}} \widetilde{y}\right\}}{2 \pi \sqrt{1-\delta^{2}}} \Psi(\widetilde{x}, \tilde{y}) d \tilde{y} d \tilde{x}\right.}{=} \\
& -\tilde{H} \int_{-b_{1}\left(m_{1}\right)}^{\infty} \int_{-\infty}^{\frac{b-m p}{\sqrt{1-2 \bar{\rho} m+m^{2}}}} \\
& \times \frac{\exp \left\{\left(g+m \sqrt{\sigma_{V}^{2} T+m_{2} \sigma_{2}^{2}}\right) \widetilde{q}+\sqrt{1-2 \bar{\rho} m+m^{2}} \sqrt{\sigma_{V}^{2} T+m_{2} \sigma_{2}^{2}} \widetilde{r}\right\}}{2 \pi \sqrt{1-\delta^{2}}} \Lambda(\widetilde{q}, \widetilde{r}) d \tilde{r} d \widetilde{q} .
\end{aligned}
$$

Now completing the square gives

$$
\begin{aligned}
C_{4}\left(m_{1}, m_{2}\right) & -\tilde{H} \exp \left\{\frac{\left(g+m V\left(m_{2}\right)\right)^{2}+2 \delta\left(g+m V\left(m_{2}\right)\right) \eta V\left(m_{2}\right)+\left(\eta V\left(m_{2}\right)\right)^{2}}{2}\right\} \\
& \cdot \int_{-b_{1}\left(m_{1}\right)}^{\infty} \int_{-\infty}^{\frac{b-m p}{\sqrt{1-2 \tilde{\rho} m+m^{2}}}} \frac{1}{2 \pi \sqrt{1-\delta^{2}}} \\
& \times \exp \left\{-\frac{1}{2\left(1-\delta^{2}\right)}\left[(\widetilde{q}-\tilde{P}-\delta Q)^{2}-2 \delta(\widetilde{q}-\tilde{P}-\delta Q)\right.\right. \\
& \left.\left.+\widetilde{r}-\delta \tilde{P}-Q)+(\widetilde{r}-\delta \tilde{P}-Q)^{2}\right]\right\} d \widetilde{r} d \widetilde{q} \\
= & -\tilde{H} \exp \left\{\frac{\left(g+m V\left(m_{2}\right)\right)^{2}+2 \delta\left(g+m V\left(m_{2}\right)\right) \eta V\left(m_{2}\right)+\left(\eta V\left(m_{2}\right)\right)^{2}}{2}\right\} \\
& \cdot \int_{-b_{1}\left(m_{1}\right)-\left(g+m V\left(m_{2}\right)\right)-\delta \eta V\left(m_{2}\right)}^{\infty} \int_{-\infty}^{\frac{b-m p}{\sqrt{1-2 \tilde{\rho} m+m^{2}}}-\delta\left(g+m V\left(m_{2}\right)\right)-\eta V\left(m_{2}\right)} \\
& \times \frac{1}{2 \pi \sqrt{1-\delta^{2}}} \exp \left\{-\frac{1}{2\left(1-\delta^{2}\right)}\left[\widetilde{w}^{2}-2 \delta \widetilde{w} \widetilde{z}+\widetilde{z}^{2}\right]\right\} d \widetilde{z} d \widetilde{w} \\
= & -\tilde{H} \exp \left\{\frac{\left(g+m V\left(m_{2}\right)\right)^{2}+2 \delta\left(g+m V\left(m_{2}\right)\right) \eta V\left(m_{2}\right)+\left(\eta V\left(m_{2}\right)\right)^{2}}{2}\right\} \\
& \cdot N_{2}\left(d_{1}\left(m_{1}, m_{2}\right), d_{2}\left(m_{1}, m_{2}\right),-\delta\right),
\end{aligned}
$$

where

$$
\begin{aligned}
d_{1}\left(m_{1}, m_{2}\right)= & b_{1}\left(m_{1}\right)+\left(g+m \sqrt{\sigma_{V}^{2} T+m_{2} \sigma_{2}^{2}}\right)+\delta \sqrt{1-2 \bar{\rho} m+m^{2}} \sqrt{\sigma_{V}^{2} T+m_{2} \sigma_{2}^{2}} \\
d_{2}\left(m_{1}, m_{2}\right)= & -b_{2}\left(m_{1}, m_{2}\right)-\delta\left(g+m \sqrt{\sigma_{V}^{2} T+m_{2} \sigma_{2}^{2}}\right) \\
& -\sqrt{1-2 \bar{\rho} m+m^{2}} \sqrt{\sigma_{V}^{2} T+m_{2} \sigma_{2}^{2}} .
\end{aligned}
$$




\section{Acknowledgements}

The authors are grateful to anonymous referees for their excellent suggestions, which greatly improved the presentation of the paper. QZ is partly supported by the National Natural Science Foundation of China [grant number 11471051], [grant number 11871010]. WW is partly supported by the National Social Science Foundation of China [grant number 16ZDA033].

\section{Funding}

QZ is partly supported by the National Natural Science Foundation of China [grant number 11471051], [grant number 11871010]. WW is partly supported by the National Social Science Foundation of China [grant number 16ZDA033].

\section{Competing interests}

The authors declare that there is no conflict of interests.

\section{Authors' contributions}

All authors contributed equally to the writing of this paper. All authors read and approved the final manuscript.

\section{Author details}

${ }^{1}$ School of Science, Beijing University of Posts and Telecommunications, Beijing, China. ${ }^{2}$ School of Banking and Finance, University of International Business and Economics, Beijing, China.

\section{Publisher's Note}

Springer Nature remains neutral with regard to jurisdictional claims in published maps and institutional affiliations.

Received: 11 April 2018 Accepted: 3 December 2018 Published online: 14 December 2018

\section{References}

1. Black, F., Scholes, M.: The pricing of options and corporate liabilities. J. Polit. Econ. 81, 637-654 (1973)

2. Merton, R.C.: Theory of rational option pricing. Bell J. Econ. Manag. Sci. 4, 141-183 (1973)

3. Johnson, H., Stulz, R.: The pricing of options with default risk. J. Finance 42, 267-280 (1987)

4. Niu, H., Wang, D.: Pricing vulnerable options with correlated jump-diffusion processes depending on various states of the economy, Quant. Finance (2016). https://doi.org/10.1080/14697688.2015.1090623

5. Hull, J., White, A.: The impact of default risk on the prices of options and other derivative securities. J. Bank. Finance 19, 299-322 (1995)

6. Jarrow, R.A., Turnbull, S.M.: Pricing derivatives on financial securities subject to credit risk. J. Finance 50, 53-85 (1995)

7. Klein, P.C.: Pricing Black-Sholes options with correlated credit risk. J. Bank. Finance 20, 1211-1229 (1996)

8. Klein, P.C., Inglis, M.: Valuation of European options subject to financial distress and interest rate risk. J. Deriv. 6, 44-56 (1999)

9. Hung, M., Liu, Y.H.: Pricing vulnerable options in incomplete markets. J. Futures Mark. 25, 135-170 (2005)

10. Klein, P.C., Inglis, M.: Pricing vulnerable European options when the option's payoff can increase the risk of financial distress. J. Bank. Finance 25, 993-1012 (2001)

11. Tian, L., Wang, G., Wang, X., Wang, Y.: Pricing vulnerable options with correlated credit risk under jump-diffusion processes. J. Futures Mark. 34(10), 957-979 (2014)

12. Yang, S., Lee, M., Kim, J.: Pricing vulnerable options under a stochastic volatility model. Appl. Math. Lett. 34, 7-12 (2014)

13. Yoon, J., Kim, J.: The pricing of vulnerable options with double Mellin transforms. J. Math. Anal. Appl. 422, 838-857 (2015)

\section{Submit your manuscript to a SpringerOpen ${ }^{\circ}$ journal and benefit from:}

- Convenient online submission

- Rigorous peer review

Open access: articles freely available online

- High visibility within the field

- Retaining the copyright to your article

Submit your next manuscript at $\gg$ springeropen.com 